\title{
Homogeneity of Shell gas stations connected to the Shell Box
}

\author{
Nonato, L. G. ${ }^{1} \dagger$, Contreras, R. C. ${ }^{1}$, Barbosa, A. O. ${ }^{1}$, Barbosa, S. \\ S. ${ }^{1}$, Basilio, J. C. C. ${ }^{1}$, Batista, J. H. ${ }^{1}$, De Sena, J. G. S. ${ }^{1}$, dA Silva, C. E. ${ }^{1}$, da \\ Silva, P. H. F. ${ }^{1}$, Godoy, C. ${ }^{1}$, Lauretto, M. ${ }^{2}$, Louzada, $\mathrm{F}^{1}$, Milani, E. \\ A. ${ }^{1}$, Oliveira, H. T. M. ${ }^{2}$, Oliveira, K. I. M. ${ }^{2}$, Ortigossa, E. ${ }^{1}$, Orenstain, \\ P. ${ }^{1}$, Silva, J. R. ${ }^{1}$,Silva, J. A. S. ${ }^{2}$, Tojeiro, C. A. V. ${ }^{1}$, and Vieira, T. ${ }^{2}$ \\ ${ }^{1}$ University of São Paulo, São Carlos - São Paulo, Brazil \\ ${ }^{2}$ Federal University of Alagoas, Maceió, Brazil
}

(Communicated to MIIR on 28 June 2021)

Study Group: VI Brazilian Study Group with Industry, São Carlos, 22-26 March 2021.

Communicated by: Luis Gustavo Nonato.

Industrial Partner: Raízen.

Presenter: Nilton Kazuyuki Ueda.

Team Members: Adriano Oliveira Barbosa, University of São Paulo, Brazil; Carlos Eduardo da Silva, University of São Paulo, Brazil; Caroline Godoy, University of São Paulo, Brazil; Cynthia A. V. Tojeiro, University of São Paulo, Brazil; Eder A. Milani, University of São Paulo, Brazil; Evandro Ortigossa, University of São Paulo, Brazil; Francisco Louzada, University of São Paulo, Brazil; Hugo Thallys Martins Oliveira, University of São Paulo, Brazil; Luis Gustavo Nonato, University of São Paulo, Brazil; Jairon Henrique Batista, University of São Paulo, Brazil; Jeann da Rocha Silva, University of São Paulo, Brazil; José Augusto dos Santos Silva, University of São Paulo, Brazil; José Guilherme Santana de Sena, University of São Paulo, Brazil; Julio Cesar de Castro Basilio, University of São Paulo, Brazil; Krerley Irraciel Martins Oliveira, University of São Paulo, Brazil; Marcelo Lauretto, University of São Paulo, Brazil; Paulo Henrique Ferreira da Silva, University of São Paulo, Brazil; Paulo Orenstain, University of São Paulo, Brazil; Rodrigo Colnago Contreras, University of São Paulo, Brazil; Suêde Santos Barbosa, University of São Paulo, Brazil; Thales Vieira, Federal University of Alagoas, Maceió, Brazil.

Industrial Sector: Energy/Utilities.

Tools: Principal Component Analysis, Survival Models, Risk Analysis, Deep Neural Network, K-Means, and Visualization Tools in Python.

Key Words: Visualization; Data Analysis; Deep Learning; Pattern Recognition; Multivariate analysis.

$\dagger$ Corresponding Author: gnonato@icmc.usp.br 
MSC2020 Codes: 68T07; 68T09; 68P05; 68N17; 68T01 (see MSC2020).

\section{Summary}

Raízen is a Brazilian company focused on energy production and distribution. Though their Shell Box app, it offers a fully digital experience for refueling and other services available in the Shell Select convenience stores. However, only $10 \%$ the current sales occur through the app, and only $20 \%$ of the units are responsible for $80 \%$ of the transactions. In this problem, we seek to better understanding this disparity through visual and statistical data exploration, classical pattern recognition approaches, and by employing Natural Language Processing algorithms to analyze user reviews.

\section{Problem Description and Approaches}

The Shell Box app is a B2B2C platform of Raízen - a Brazilian company focused on energy production and distribution. The app delivers a fully digital experience for refueling and other services available in the Shell Select convenience stores. Currently, more than 3,500 Shell gas stations are available for the Shell Box experience in Brazil.

Unfortunately, Shell Box sales represent merely 10\% of the total Shell gas stations' sales. Besides, $80 \%$ of such transactions are concentrated in only $20 \%$ of the gas stations.

\subsection{Problem Statement}

Given the aforementioned disparity, the following initial question were proposed to analyze this problem:

"Which factors influence each gas station performance?"

- Localization (near educational establishments, hospitals, offices?)

- State/city/town?

- Services provided by the gas station (conveniences, additional services,...)?

- Time since the Shell Box was available in the gas station?

- Number of pumps?

- Layout of the unit?

- Profile of the unit (urban/road)?

- Opening times of the gas station (such as 24h)?

- Consumer profile and demography of the region (youth, elderly, commuters,...)?

- Efficiency/excellence relation of the unit?

- Is it a single site or belongs to a network of units?

The problem was addressed by the following approaches:

(1) Statistical data exploration

(2) Visual data exploration 
(3) Classical pattern recognition

(4) Analysis of users reviews

\section{WP1 - Statistical Data Exploration}

In this section, three approaches will be taken to solve the problem of checks to increase the homogeneity of the participation of the Shell Box platform at Shell gas stations. These approaches were divided as follows:

- Gas station segmentation;

- Mixed Logistic Regression Model;

- Survival analysis from registration time to first use.

\subsection{Gas station segmentation}

As the objective of this section is to use segmentation by gas station, it was decided to apply the Principal Component Analysis (PCA) to identify these groups. This finding came after an assessment of all the bases individually, as will be seen in the next topics.

\subsubsection{Methodology}

Two steps of methodology were used to build the final results. The first is a descriptive univariate assessment for all variables in all databases, to identify possible variables that could be grouped according to the objective of the work. This descriptive analysis can be composed of graphs and/or tables whose purpose is to present a summary of what the variable represents.

The second stage, on the other hand, refers to Principal Component Analysis (PCA), which is a method of grouping variables that analyzes the interdependence of multivariate data. In case of similarities in the data set, it may be possible to explain most of the information in a smaller set of dimensions that can be again appropriately named. From the new components created and selected, according to a linear combination, it is possible to plot them in relation to the data and then determine sets with similar characteristics, that is, segmenting the data into clusters. For more information on PCA, see [1].

\subsubsection{Results}

With the analysis methodology structure in hand, the following results were obtained:

\section{Pre-processing of data}

In this item, the behavior of the variables of the 4 available databases was evaluated ("transactions.csv", "consumers.csv", "stores.csv", "pumplogs.csv") and reports were generated for each of the databases, according to Annex 1. 


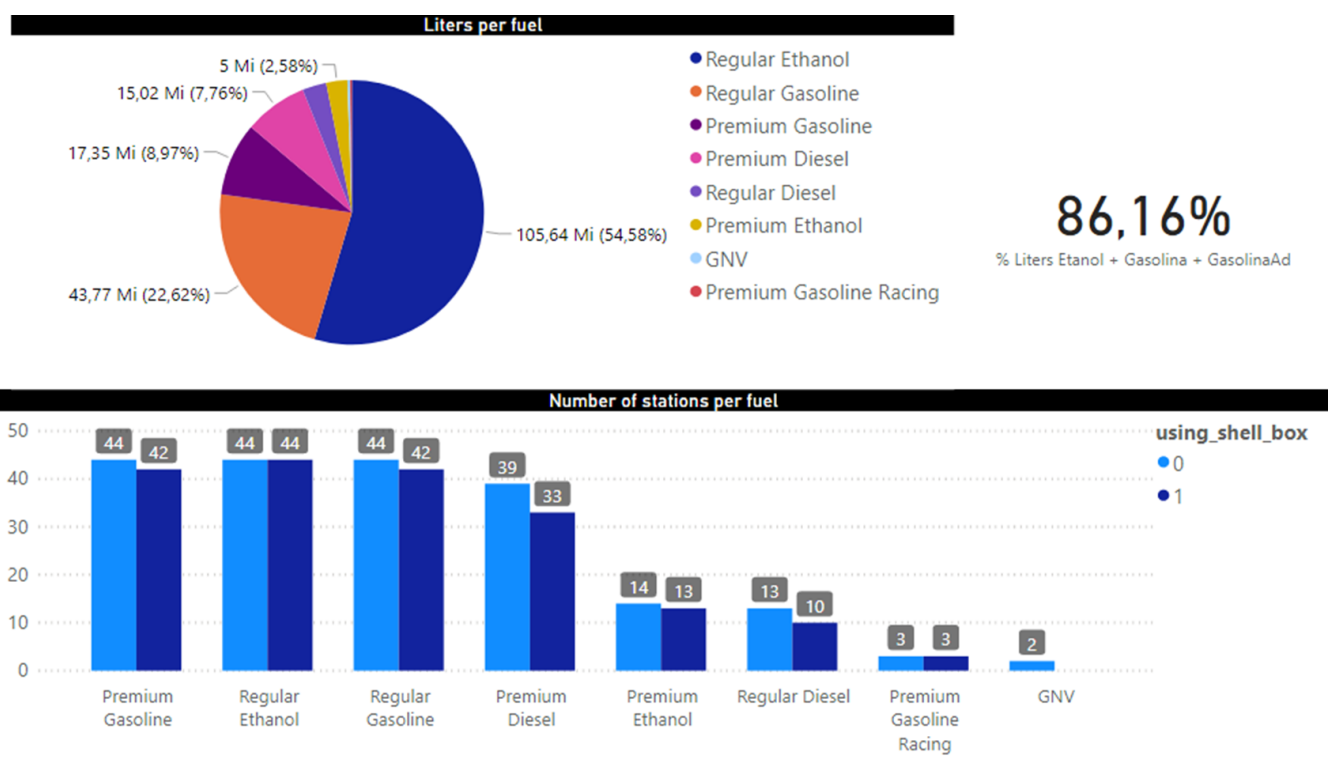

Figure 1. Distribution of number of liters and stations, both by fuel.

\section{Preparation of the database}

All available databases were used in the segmentation, however, as the main segmentation base, the "pumplogs.csv" and "stores.csv" databases were used with the store_id index (station identifier) as the indexing variable. In addition, in order to be able to capture the largest amount of accurate information, variables that had the least number of missing values were used.

\section{Application of PCA and formation of segmented groups}

The variables available for the PCA were a total of 40 variables, among them being distributed among the 8 types of fuels that Shell stations offer (Fig. 1). For each station and type of fuel, the quantity in liters using the Shell Box, the quantity in liters not using the Shell Box, the price per liter, the total number of transactions using the Shell Box and the total number of transactions not using the Shell Box were considered. In addition to two more variables: general in reais received from payments at the service stations and the total number of transactions per service station. In Figure 1, it can be seen that the highest percentage $(86.16 \%)$ of fuels at the service stations is among the fuels: regular gasoline, gasoline with additives and ethanol. Therefore, it was decided to use only these results in the calculation of the PCA. In addition, the other fuels do not have a sufficient number of data per service station that can contribute in any significant way to the results, resulting in a final number of 17 variables and 44 stations, distributed in two cities (Ribeirão Preto - 26 and Sorocaba - 18) for application of the PCA.

A verification with a Screeplot was applied, which indicated the use of 2 components to explain the data according to the 17 variables, as well as a cumulative percentage of explanation of the variables of $72.5 \%$, indicating a reasonably good value of variables explained by the two components. Table 1 shows components 1 and 2 that explain the 
Table 1. Table of the components 1 and 2 that explain each variables.

\begin{tabular}{ccc}
\hline Variables & Component 1 & Component 2 \\
\hline Common.Gasoline.sum_quantity_in_litres_using_shell_box & 0.863 & 0.32 \\
Common.Gasoline.sum_quantity_in_litres_not_using_shell_box & 0.555 & 0.751 \\
Common.Gasoline.mean_price_per_litre & -0.262 & -0.213 \\
Common.Gasoline.total_using_shell_box & 0.807 & 0.398 \\
Common.Gasoline.total_not_using_shell_box & 0.214 & 0.934 \\
Common.Ethanol.sum_quantity_in_litres_using_shell_box & 0.876 & 0.249 \\
Common.Ethanol.sum_quantity_in_litres_not_using_shell_box & 0.582 & 0.703 \\
Common.Ethanol.mean_price_per_litre & 0.887 & -0.334 \\
Common.Ethanol.total_using_shell_box & 0.366 & 0.319 \\
Common.Ethanol.total_not_using_shell_box & 0.95 & 0.908 \\
Gasoline.Additives.sum_quantity_in_litres_using_shell_box & 0.714 & 0.416 \\
Gasoline.Additives.sum_quantity_in_litres_not_using_shell_box & & \\
Gasoline.Additives.mean_price_per_litre & 0.942 & 0.157 \\
Gasoline.Additives.total_using_shell_box & 0.689 & 0.746 \\
Gasoline.Additives.total_not_using_shell_box & 0.378 \\
Total_in_reais_fuel_station & \\
Total.Transactions & & 0.625
\end{tabular}

variables. It is observed that component 1 has a greater explanation for most of the variables that concern the use of the Shell Box; component 2 has a greater explanation for most variables that do not relate to the use of the Shell Box.

In addition, as these main variables relate to fuel consumption in quantity and liters, it was decided to call them "Litration with Shell Box" and "Litration without ShellBox". In Figure 2 it is observed the crossing of the components forming quadrants that were used to classify and segment the stations into 4 groups according to their characteristics, with the points being each station fuel in the sample. In Figure 2, CS+ SS+ indicates the best of scenarios, with many stations with large fuel liters sold with and without Shell Box; CS- SS+ indicates a regular scenario in which there is a low number of service stations with Shell Box, but a high number of service stations without Shell Box; CS+ SS - indicates a regular situation, but better than the previous one, being a high number of stations with Shell Box and low number of stations without Shell Box; and, finally, CS - SS - which indicates the worst of the scenarios where there is low litigation of service stations with and without Shell Box.

\section{Segmentation considering litration and use of Shell Box}

In this stage, the results are presented from the segmentation of the gas stations in four groups considering the classification by litration with and without Shell Box. The software used for analysis was the $\mathrm{R}$ for the segmentation and the PowerBI to carry out the graphic analysis and verification of individual characteristics by group.

The first analysis to be highlighted is that of Ribeirão Preto, it is such that, although the sale in liters is high for the orange group (CS-SS+), as well as in the ideal group of stations (green, CS+ SS+), the number of campaigns carried out to attract customers is low, as well as the amount of discounts $(\mathrm{R} \$)$ offered, which goes in contradiction with the green group, whose value is high, that is, there could be some type of marketing action within the fuel stations of the orange group, to increase the number of campaigns and discounts; and attract or convert more customers in the use of the Shell Box App when using the services of the fuel station (Figure 3). 


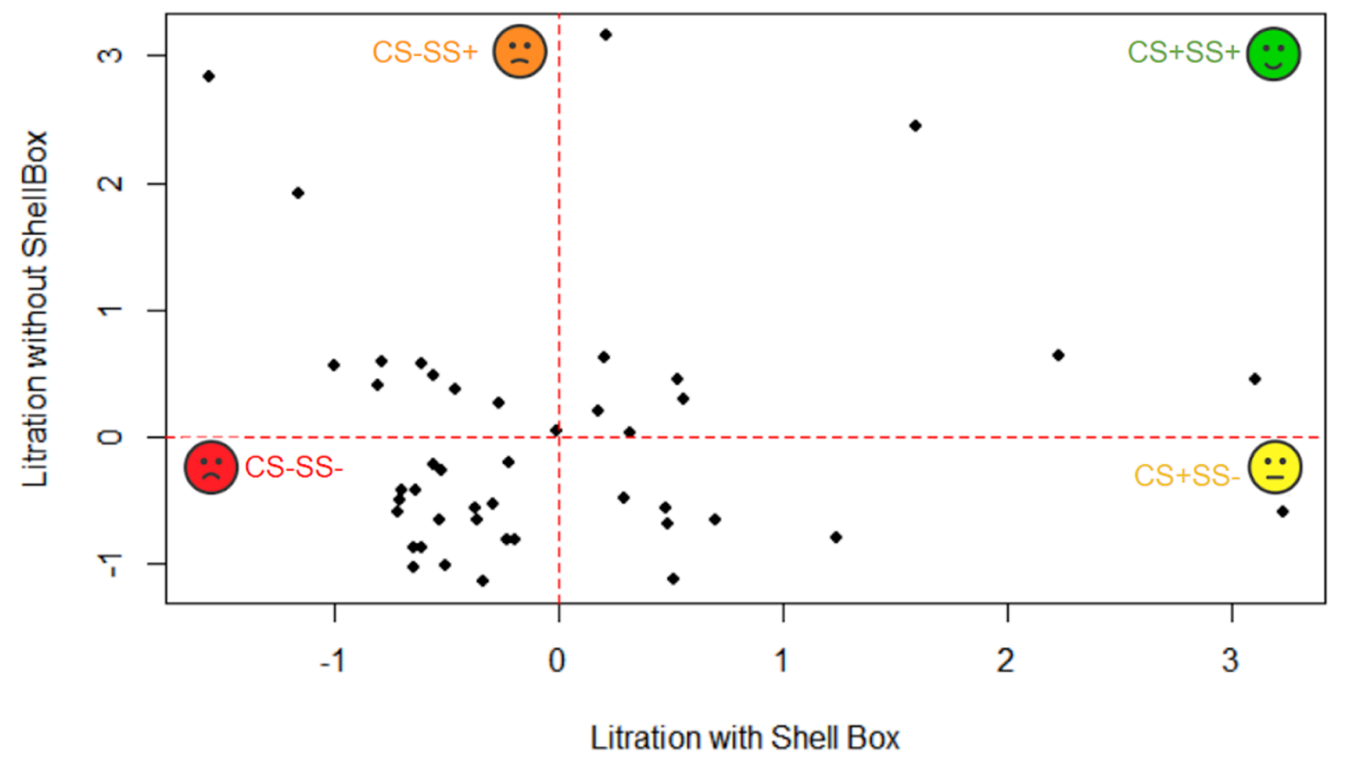

Figure 2. Litration with Shell Box versus without ShellBox and quadrants that were used to classify and segment the stations.

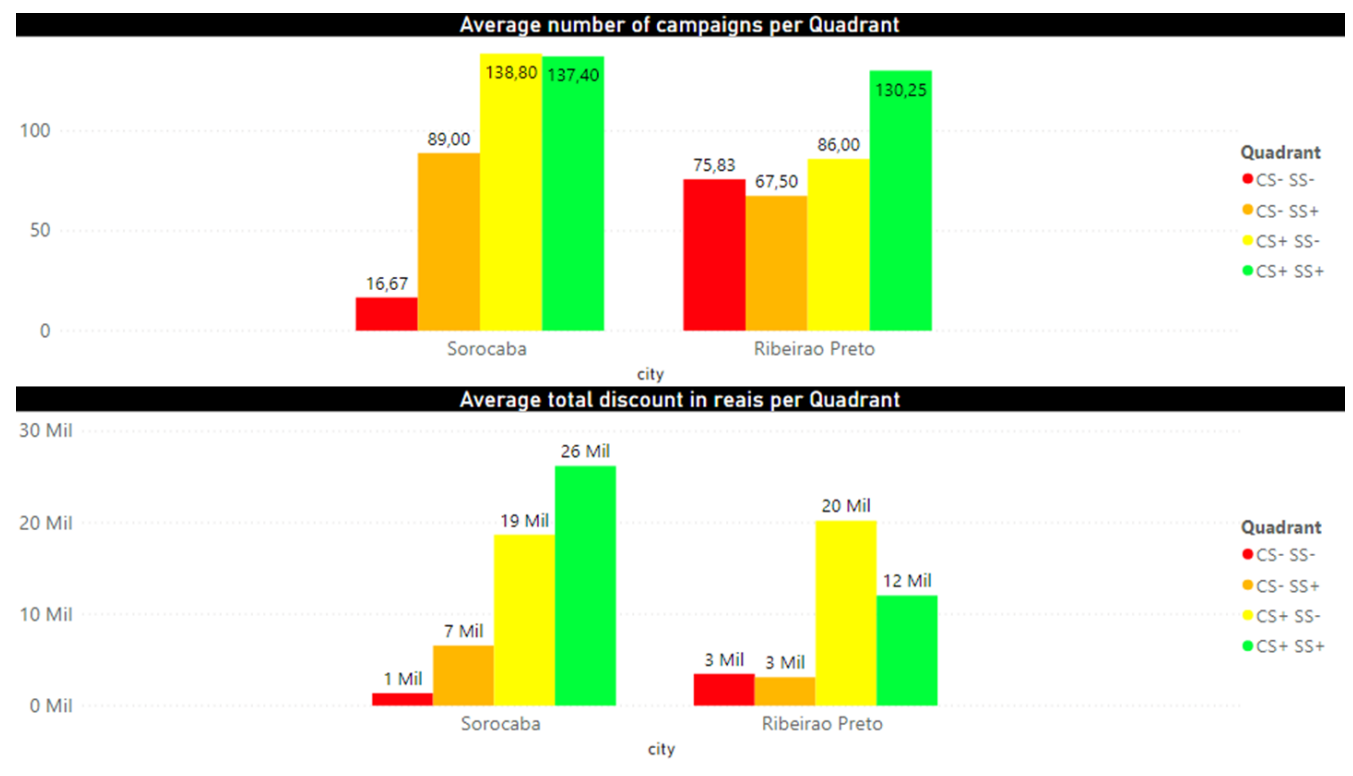

Figure 3. Distribution of the average number of campaigns and discounts by group and by city.

Still in Figure 3, considering Ribeirão Preto and comparing the behavior of the groups, it is observed that the stations in the yellow group (CS+SS-) have a high average number of campaigns and discounts offered to customers, while in the red group (CS-SS-), there is the opposite, that is, low amounts of campaign quantities and discounts. Despite being 


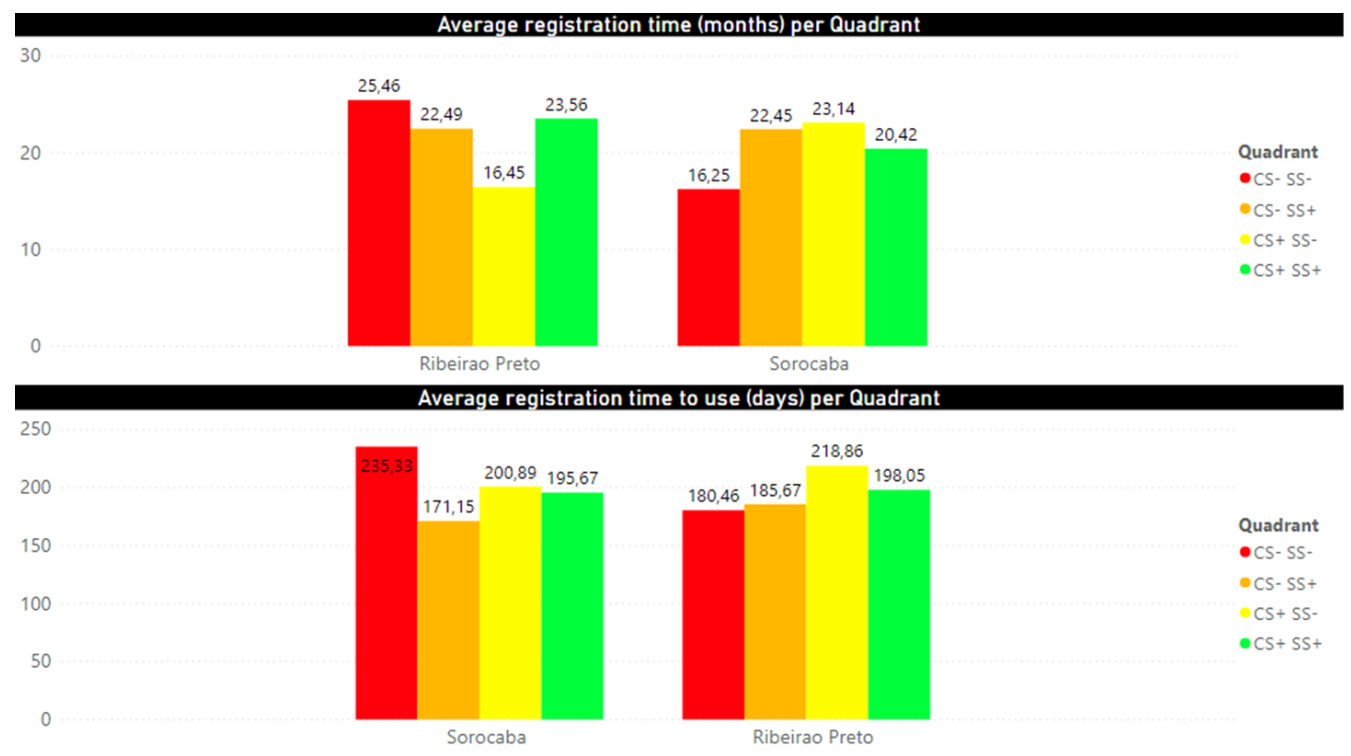

Figure 4. Average customer registration time and registration time to use by group and by city.

opposites and for that reason being in different groups, it is also observed that in the yellow group $(\mathrm{CS}+\mathrm{SS}-)$, the gas stations have a low registration time (average around 16 months) (date of the first transaction minus the date in months), that is, they are fast loyal users; for the red group (CS-SS-), this registration time is high (average around 25 months), which indicates that the low number of campaigns and discounts can influence customer engagement in the use of the Shell Box application In this case, it is possible to have some type of marketing action to increase the number of campaigns and discounts, so that the customers of the stations in the red group converge to the yellow group and then to the green group (Figures 3 and 4).

In the case of Sorocaba, other problems may be interfering in the conversion of red to yellow stations, such as the number of transactions (from medium to low), or another factor arising from other variables not studied. This is reflected in other variables such as total sales, total transactions and total consumers (see Figures 5 and 6 ) where the red group has the worst performance in these assessments.

An analysis with more variables at the customer and region level could be done to verify the reason why users of the red group ( $\mathrm{CS}-\mathrm{SS}-)$ did not use the Shell Box for longer periods of time.

The last type of analysis addressed, which was selected as interesting to highlight, was with the use of the geolocation of the gas stations of each of the 4 segmented groups. Figure 7 shows the Sorocaba map with the colored marking of the locations of each gas station and the type of group. From it, it is possible to verify that the green group fuel stations (high use of Shell Box and high quantity of liters sold), which are the ones with the best performance, are located close to important highways and towards the city. In addition, the gas stations that are in the direction of entry are those of the red group and the fuel stations of the yellow group are all concentrated in the center of the city. A 


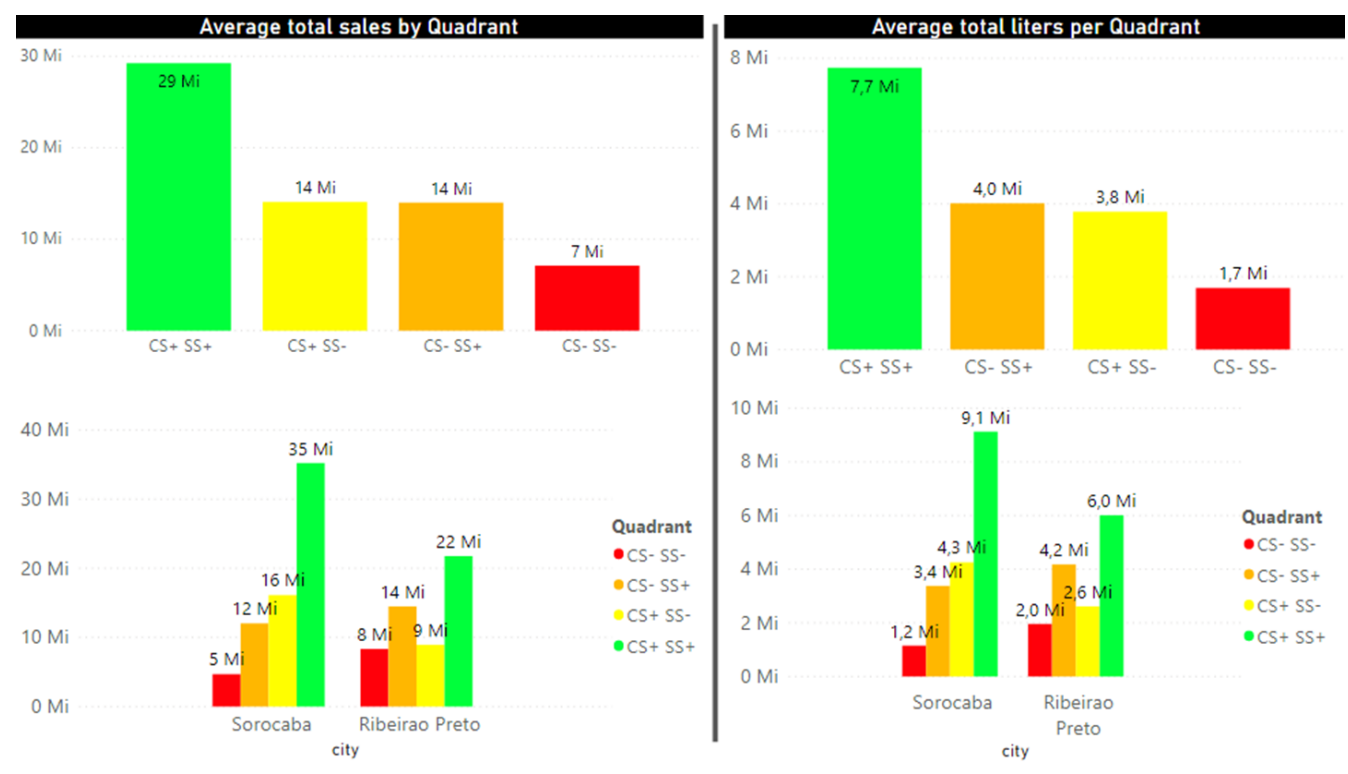

Figure 5. Average of total sales and total liters by group and by city.

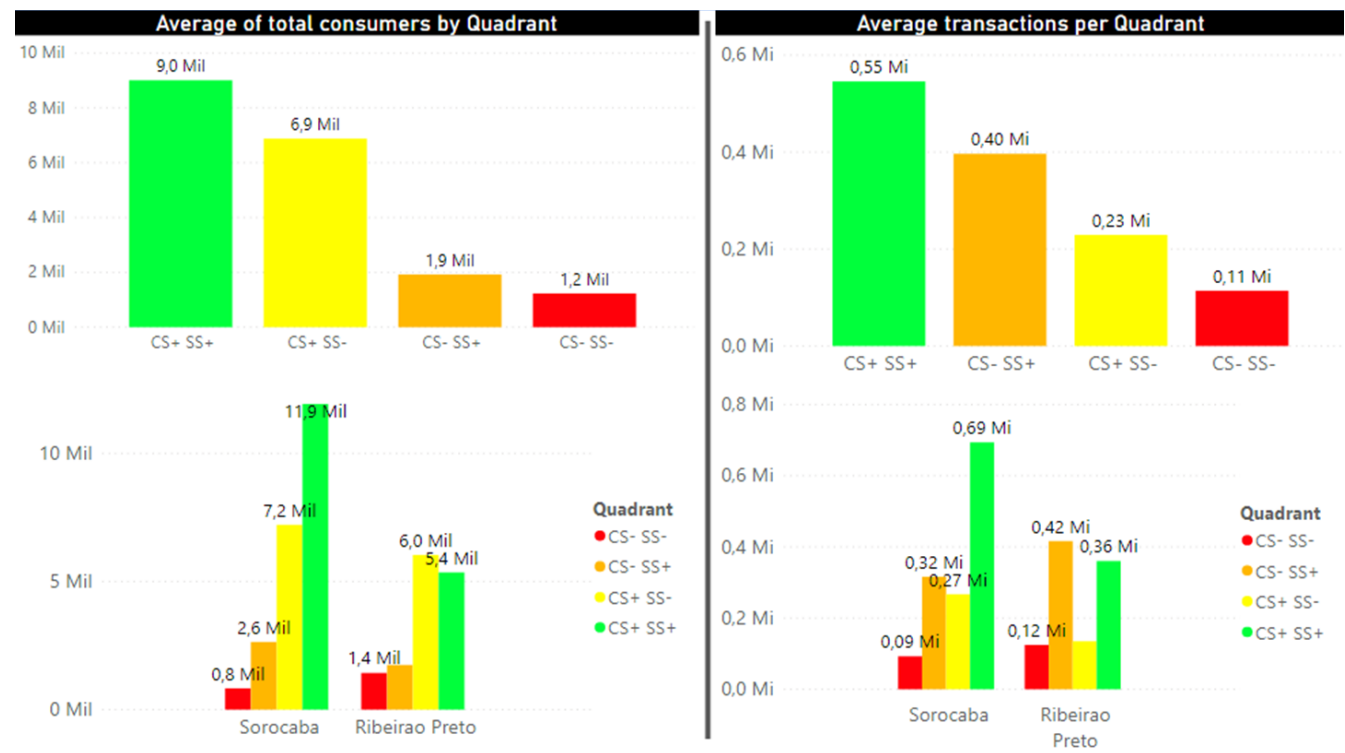

Figure 6 . Average of consumers and transactions by group and by city.

highlight for the fuel station of the orange group in the upper right corner of the map, as it is close to the highway and has a good amount of liters sold, however it does not have a good use of the Shell Box. Therefore, a more detailed analysis of this station can generate an action to increase sales in the Shell Box. The map was also made for the city of Ribeirão Preto, however, as a more complex result was obtained, the analyzes for it need more time and more variables to identify the characteristics location. 


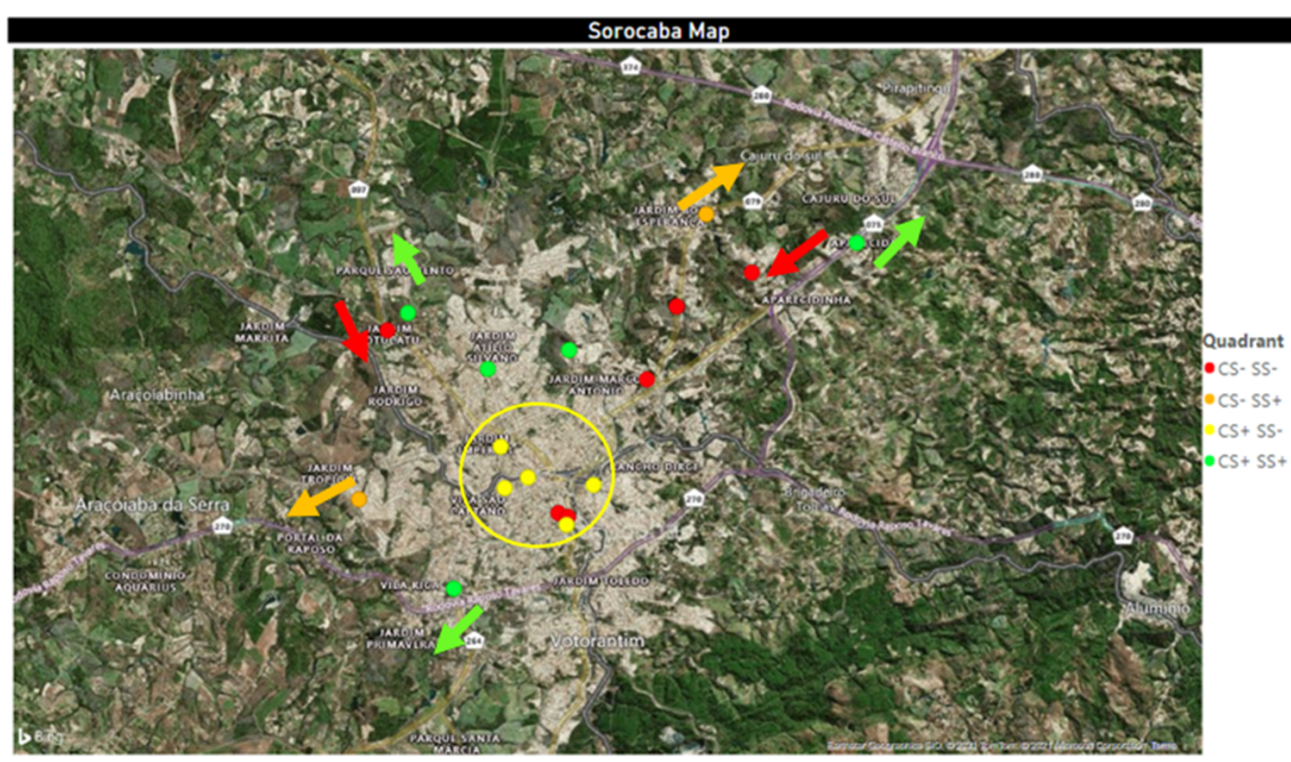

Figure 7. Sorocaba map with distribution of the gas stations by group.

\subsubsection{Conclusions and Proposition of actions}

In this section, there are some important information and observations regarding the proposed actions and insights found during the analysis. To this end, the SWOT Matrix management tool was used, which is widely used by managers to indicate strengths, weaknesses, opportunities and threats in project planning, but which can be adapted to different situations, such as this case, in which the matrix will indicate topics and proposition of actions.

\subsection{Mixed logistic regression model}

In this section, we aim to predict the transactions that will be executed using the Shell Box app. For this, we will consider a classification model based on the mixed logistic regression. This statistical technique is widely applied to analyze clustered binary data. In our work, each cluster represents a different consumer, who can perform several transactions via the app during a certain period. Then, we will use a two-level logistic model with a random intercept. Further details on this relevant statistical technique can be found in [4], among others.

In this application, we consider only part of the original database, consisting of $n=$ 4,000 transactions performed during the two-year period of 2019-2020 in Sorocaba city, Brazil. This dataset is balanced, that is, it is composed of 2,000 transactions using the app, and 2,000 transactions not using Shell Box.

First, we split our data into two subsets: training data (70\%) and testing data (30\%). Then, the mixed logistic regression model is fitted to the training data considering the following variables available in the original document: 
Table 2. Table of SWOT Matrix with indicate strengths, weakness, opportunities and threats, and proposition of actions.

\begin{tabular}{|c|c|c|}
\hline SWOT Analysis & Strengths & Weakness \\
\hline \multirow[t]{4}{*}{ Opportunities } & $\begin{array}{l}\Rightarrow \text { Well-targeted groups } \\
\text { in terms of consumption } \\
\text { in liters, total paid and } \\
\text { number of different transac- } \\
\text { tions. }\end{array}$ & $\begin{array}{l}\Rightarrow \text { Conversion work for stations in the orange } \\
\text { to green groups (Ribeirão Preto) and from red }\end{array}$ \\
\hline & $\begin{array}{l}\Rightarrow \text { Provides a more focused } \\
\text { focus on conversion stations. }\end{array}$ & to yellow groups (Ribeirão Preto and Sorocaba) \\
\hline & $\begin{array}{l}\Rightarrow \text { Possibility of elaborating } \\
\text { a system of visualization of }\end{array}$ & $\begin{array}{l}\text { with a focus on increasing the number of } \\
\text { discounts and increasing campaigns }\end{array}$ \\
\hline & $\begin{array}{l}\text { results in real time, linking } \\
\text { automatically with the } \\
\text { database. }\end{array}$ & at these specific stations. \\
\hline Threats & $\begin{array}{l}\Rightarrow \text { Very important data from } \\
\text { the bases, but they need to be } \\
\text { fine-tuned in the collection } \\
\text { to avoid misleading data, } \\
\text { such as wrong birth dates. } \\
\text { This could influence the detec- } \\
\text { tion of problems in an } \\
\text { automatic verification system, } \\
\text { for example. }\end{array}$ & $\Rightarrow$ Incomplete data precluding further analysis. \\
\hline
\end{tabular}

- "using_shell_box": binary response variable (1 - Yes, 0 - No);

- "shell_select";

- "segment": 4-level covariate (1 - Segment 1, 2 - Segment 2, 3 - Segment 3, 4 - Segment $4)$;

- "pandemics": pandemics indicator (1 - Yes, 0 - No);

- "consumer_id": random effect.

It is worth mentioning that the covariate "segment" is the same one as previously described (i.e., created using the Principal Component Analysis (PCA) multivariate technique). We also consider that Coronavirus Disease (COVID-19) pandemics started in Brazil in March 2020. Finally, it is important to note that the consumer identification ("consumer_id" variable) is only available (i.e., with values different from zero or NA "not available") in the original database for consumers whose transactions were carried out with the app. Thus, for the transactions not using Shell Box, we assumed that they were performed by different consumers.

Our proposed model can be fitted using the glmer(., family="binomial") function of the $\mathrm{R}$ package lme4 [2]. The maximum likelihood estimation results are presented in Table 3. Note that the "shell_select" covariate has no significant effect on the response of interest. This table also shows the odds ratios for all covariates, which reveals, among others, that the chances of transaction using Shell Box are almost three times higher on pandemic times.

In order to assess the predictive performance of the fitted model, we calculate the following evaluation metrics from the testing data: accuracy (ACC), sensitivity (SEN), specificity (SPE), positive and negative predictive values (PPV and NPV, respectively), Matthews correlation coefficient (MCC), and F1-score. In addition, we build the receiver 
Table 3. Estimation results of the mixed logistic regression model fitted to the Sorocaba dataset $(n=2,800)$. OR $=$ Odds Ratio, $\mathrm{SE}=$ Standard Error of the Estimate.

\begin{tabular}{|c|c|c|c|c|c|}
\hline Coefficient (Fixed-effects) $*$ & Estimate & OR & SE & $\mathrm{z}$ value & $\operatorname{Pr}(>|\mathbf{z}|)$ \\
\hline Intercept & -0.622 & - & 0.096 & -6.452 & $<0.001$ \\
\hline shell_select - 1 & -0.190 & 0.827 & 0.123 & -1.549 & 0.121 \\
\hline segment - 2 & -0.810 & 0.445 & 0.184 & -4.395 & $<0.001$ \\
\hline segment - 3 & -1.658 & 0.191 & 0.228 & -7.288 & $<0.001$ \\
\hline segment - 4 & 0.608 & 1.836 & 0.114 & 5.346 & $<0.001$ \\
\hline pandemics - 1 & 1.074 & 2.926 & 0.111 & 9.672 & $<0.001$ \\
\hline
\end{tabular}

* Random-effects: Variance (intercept) $=1.035$.

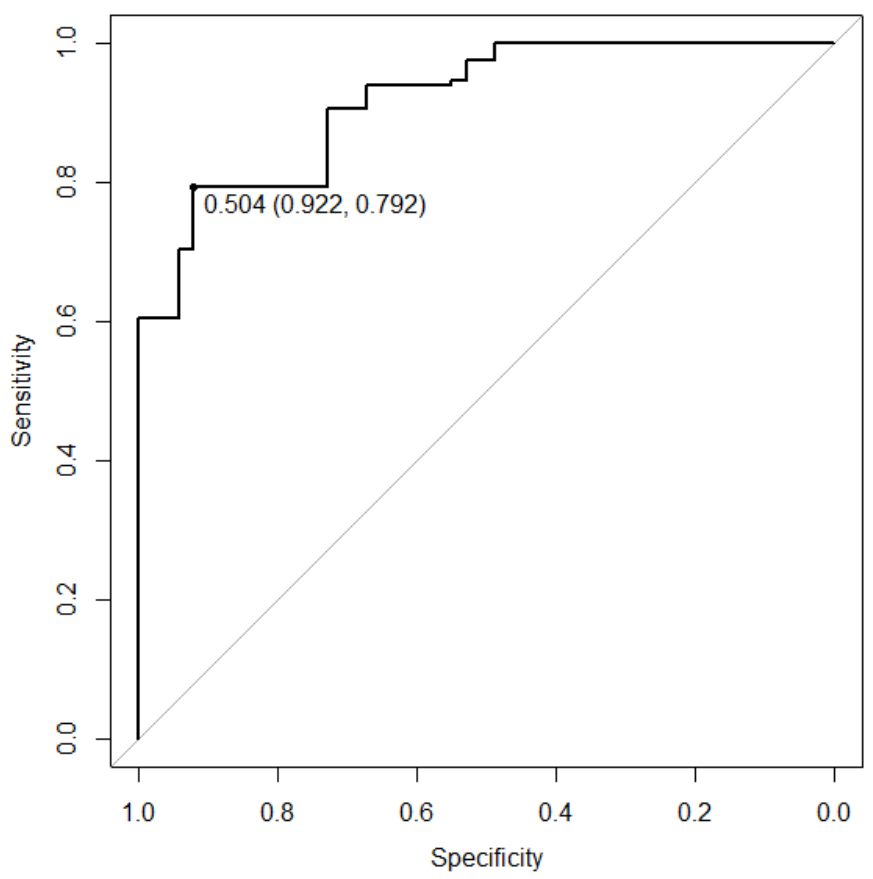

Figure 8. ROC curve for Sorocaba $(n=2,800)$.

Table 4. Predictive performance of the mixed logistic regression model fitted to the Sorocaba dataset $(n=1,200)$.

\begin{tabular}{ccccccc} 
ACC & SEN & SPE & PPV & NPV & MCC & F1 \\
0.644 & 0.622 & 0.667 & 0.662 & 0.628 & 0.290 & 0.641 \\
\hline
\end{tabular}

operating characteristic (ROC) curve (SPE versus $\mathrm{SPE}$ ), but from the training data. The obtained results indicate a good performance of the proposed mixed logistic regression model (see Figure 8 and Table 4).

Finally, we split the testing data into three groups, according to the estimated probabilities of transaction using Shell Box (scores). These groups should contain roughly the 
Table 5. Scores ordering and accumulated percentage of transactions using Shell Box, considering the mixed logistic regression model fitted to the Sorocaba dataset $(n=1,200)$.

\begin{tabular}{cccc}
\hline Group ${ }^{*}$ & $\mathbf{n}$ & \% using_shell_box & Accumulated \% using_shell_box \\
\hline G1 & 215 & 28.84 & 100.00 \\
G2 & 756 & 49.89 & 89.90 \\
G3 & 229 & 76.42 & 28.50 \\
\hline * G1: score $<0.373 ;$ G2: $0.373 \leq$ score $\leq 0.623 ;$ G3: score $>0.623$.
\end{tabular}

Table 6. Estimation results of the mixed logistic regression model fitted to the Ribeirão Preto dataset $(n=2,800)$. OR $=$ Odds Ratio, $\mathrm{SE}=$ Standard Error of the Estimate.

\begin{tabular}{cccccc}
\hline Coefficient (Fixed-effects) & Estimate & OR & SE & $\mathbf{z}$ value & $\operatorname{Pr}(>|\mathbf{z}|)$ \\
\hline (Intercept) & -0.365 & - & 0.156 & -2.346 & 0.019 \\
shell_select - 1 & 0.982 & 2.671 & 0.200 & 4.903 & $<0.001$ \\
shell_select - 2 & 0.402 & 1.495 & 0.142 & 2.830 & 0.005 \\
segment - 2 & -1.491 & 0.225 & 0.196 & -7.599 & $<0.001$ \\
segment - 3 & -0.166 & 0.847 & 0.152 & -1.098 & 0.272 \\
segment - 4 & 1.877 & 6.535 & 0.276 & 6.809 & $<0.001$ \\
pandemics - 1 & 0.819 & 2.268 & 0.134 & 6.117 & $<0.001$ \\
\hline * Random-effects: Variance (intercept) $=1.330$. & & &
\end{tabular}

${ }^{*}$ Random-effects: Variance (intercept) $=1.330$.

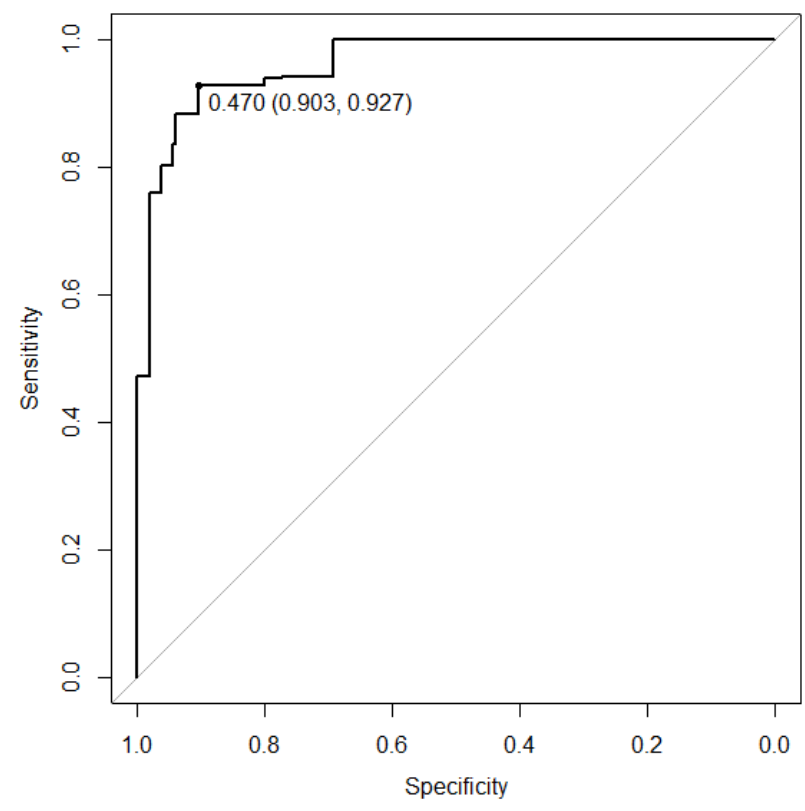

Figure 9. ROC curve for Ribeirão Preto $(n=2,800)$.

same frequency of consumers. For all groups, we calculate the observed app transactions rates, as well as the accumulated rates. These results are summarized in Table 5 .

We also perform a similar analysis (i.e., with the same previous steps) for the Ribeirão Preto city, Brazil. The results are exhibited below (see Tables 6-8 and Figure 9), and also show the adequate performance of the proposed classification model. 
Table 7. Predictive performance of the mixed logistic regression model fitted to the Ribeirão Preto dataset $(n=1,200)$.

\begin{tabular}{ccccccc}
\hline ACC & SEN & SPE & PPV & NPV & MCC & F1 \\
\hline 0.678 & 0.573 & 0.787 & 0.735 & 0.641 & 0.368 & 0.644 \\
\hline
\end{tabular}

Table 8. Scores ordering and accumulated percentage of transactions using Shell Box, considering the mixed logistic regression model fitted to the Ribeirão Preto dataset $(n=1,200)$.

\begin{tabular}{cccc}
\hline Group $*$ & n & \% using_shell_box & Cumulative \% using_shell_box \\
\hline G1 & 387 & 23.25 & 100.00 \\
G2 & 445 & 53.48 & 85.22 \\
G3 & 368 & 76.35 & 46.14 \\
\hline * G1: score $<0.319 ;$ G2: & $0.319 \leq$ score $\leq 0.645 ;$ G3: score $>0.645$.
\end{tabular}

\subsection{Survival analysis model}

The purpose of this section is to identify possible relationships between the time spent by the customer between registering and making the first purchase, using the Shell Box. To do this, we calculate the difference between two variables in the "consumers" dataset, which are "first_transaction" and "created_at". The end result is to obtain the number of registration days until the first purchase is made.

In the preliminary analysis of the variable created, we identified that 684 registrations had a negative date, that is, the date of the first purchase is prior to the registration date, thus indicating an error in the registration. We decided to remove these records from the database. The summary of the created variable is shown in Figure 10.

We note that $34.8 \%$ of customers make their first purchase on the same day of registration, about $71.4 \%$ of customers take up to one month from registration until their first purchase.

We noticed that all registered customers presented the date of the first purchase, this on the basis provided. But we believe that this does not happen in the company's daily routine. Therefore, we adopted the Survival/Reliability Analysis techniques to perform the data analysis. For more details, see [3] and [7].

The dataset presents customers who registered until the year 2021, consequently, there is no reasonable follow-up period for them. Therefore, we have adopted that customers with lifetime over one year will have their time censored on the right.

We started the analysis by estimating the hazard function, which can be interpreted as the instant rate of the first purchase. In Figure 11, we notice that the first day has the highest rate, decreasing until around 80 days, and after that it remains constant. In the sequence we estimate the survival function, see Figure 3. We noticed a decrease in the first 30 days, and after this time, the function shows a very slight, almost constant decay. The median time is equal to 4 days, that is, half of the customers take up to 4 days to make the first purchase after completing the registration.

In the sequence we estimate the survival function, see Figure 12. We noticed a decrease in the first 30 days, and after this time, the function shows a very slight, almost constant 


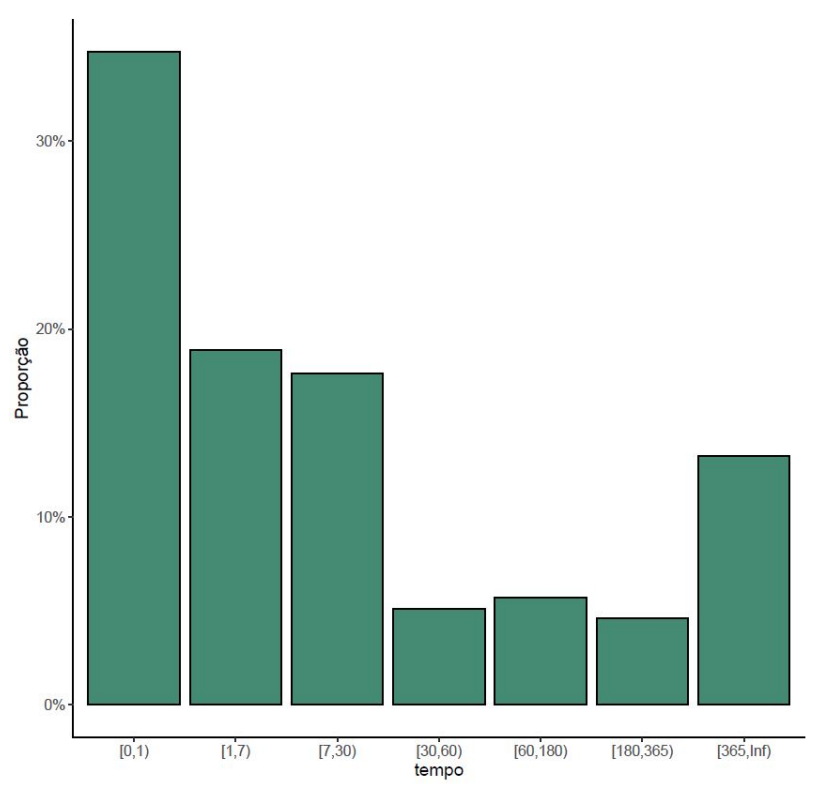

Figure 10. Summary of time until activation.

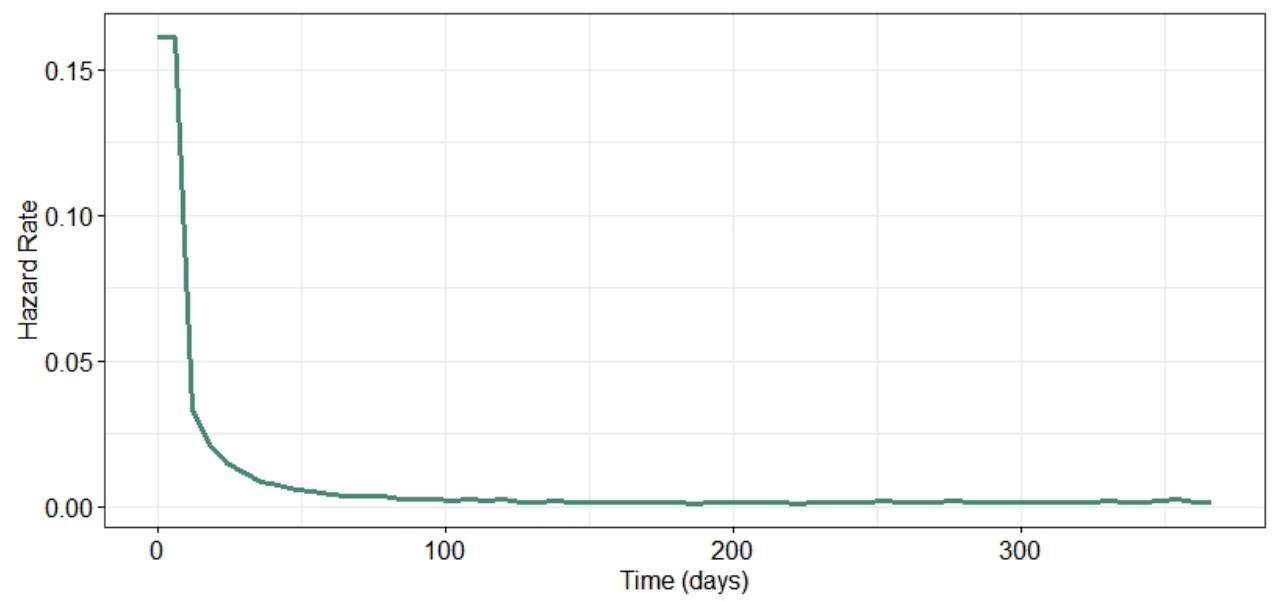

Figure 11. Empirical estimation of the hazard function

decay. The median time is equal to 4 days, that is, half of the customers take up to 4 days to make the first purchase after completing the registration.

Using the "id_cliente" key, we build two variables from the "transactions" data set, they are

- preferred fuel: obtained from the most frequent fuel in the supply with Shell Box. In this analysis, we observe only the fuels gasoline, gasoline additive and ethanol.

- discount utilization: from the campaign identification variable, it is verified if there was 


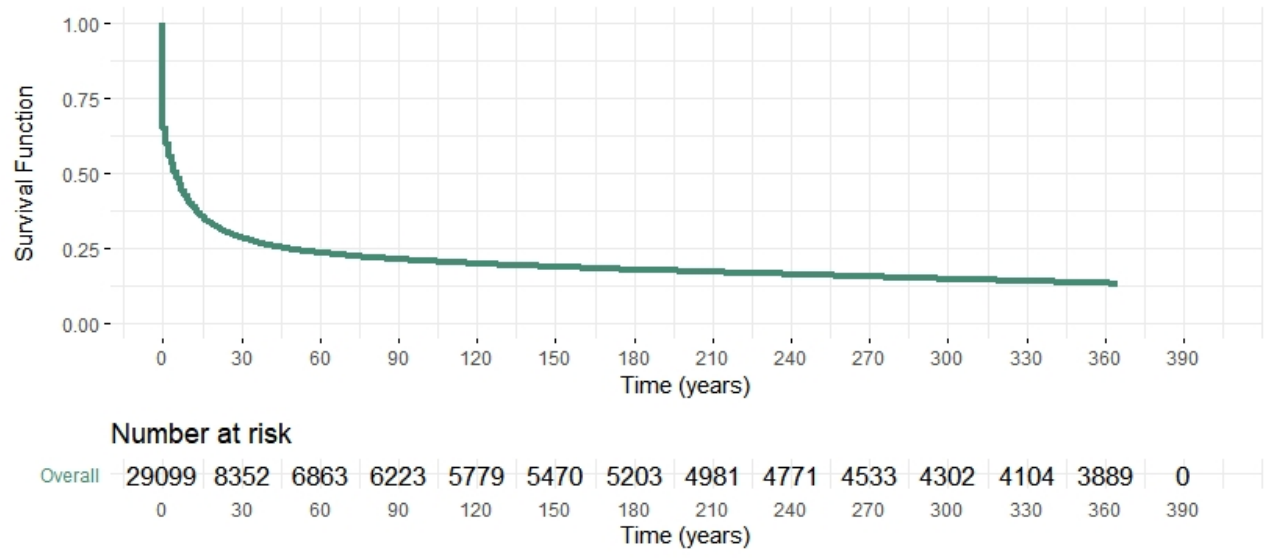

Figure 12. non-parametric estimation of the survival function

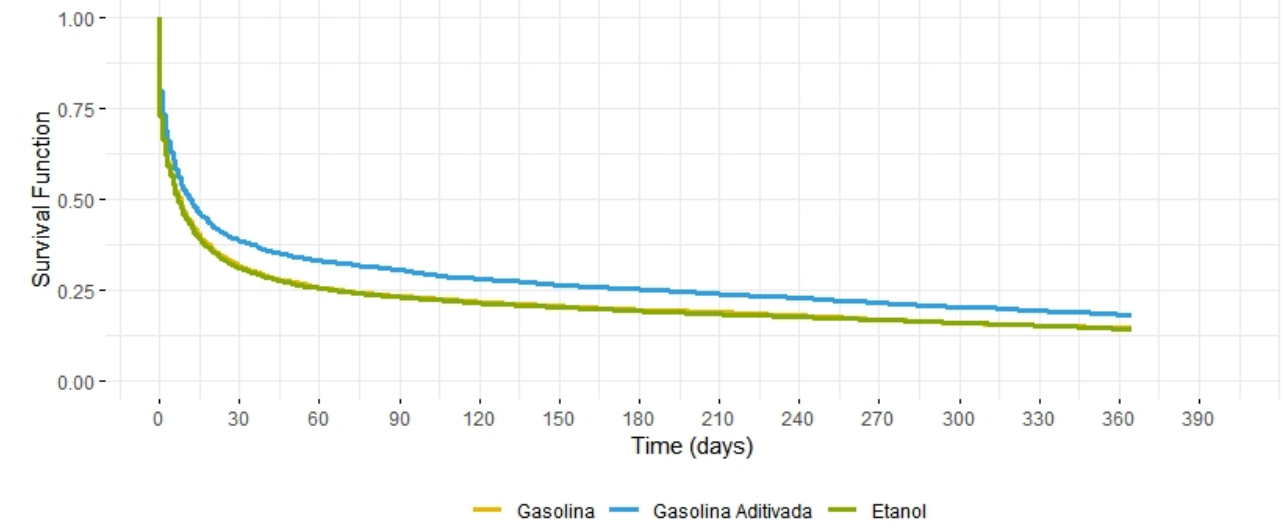

Figure 13. Non-parametric estimation of the survival function, considering the preferred fuel

any supply with a discount in the observed time. If there is no discount, the created variable receives a value of zero, otherwise, we assign a value of 1 .

From these two variables, we observe the behavior of the survival function. In Figure 13, we notice that gasoline and ethanol fuels have similar behaviors, throughout the analyzed time. However, consumers who have a greater preference for gasoline additive take longer to make their first purchase. Since the median time is equal to 4, 6 and 11, for gasoline, ethanol and gasoline additive. Therefore, among customers who prefer gasoline additive, half of them take up to 11 days to make their first purchase, a number of days 3 times greater when compared to gasoline and 2 times greater when compared to ethanol.

In Figure 14, we observe the survival function considering the discount variable. We observed a significant difference between the curves. Those customers who used a discount have a shorter time until the first purchase. The median time is 3 and 10, with and without 


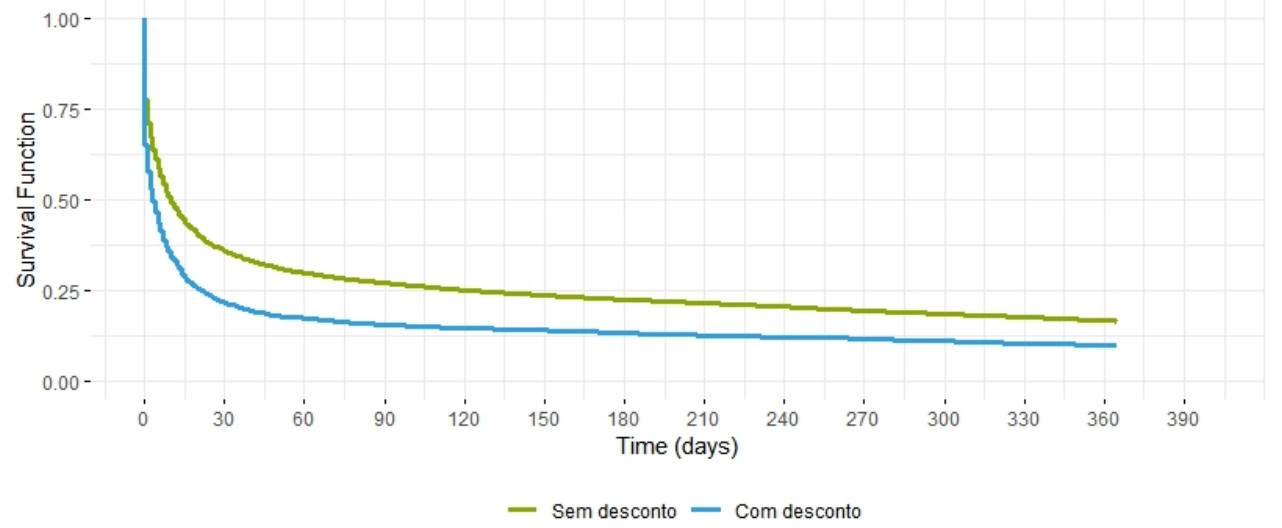

Figure 14. Non-parametric estimation of the survival function, considering the discount utilization

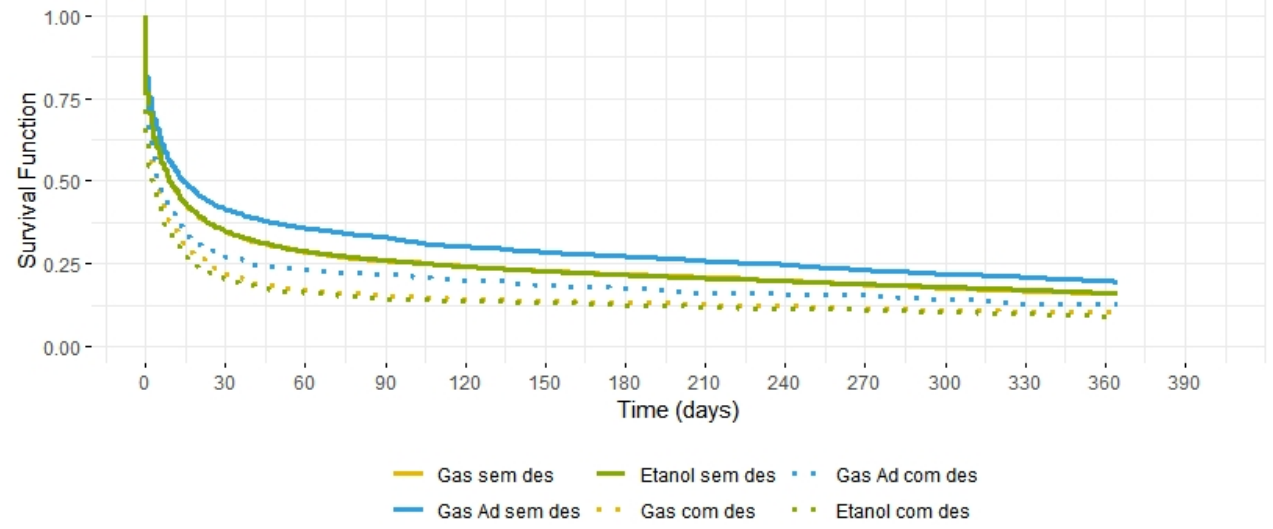

Figure 15. Non-parametric estimation of the survival function, considering the preferred fuel and the discount

discount, respectively. Therefore, the time until the first purchase of customers who do not use a discount is three times the time of those who use a discount.

Finally, in Figure 15, we estimate the survival function considering both created variables. We note that the behavior of customers who do not use a discount and refuel with gasoline additive has the longest median time, which is 14 days, seven times longer than customers who refuel with ethanol and used a discount at least once.

From the above, we noticed that almost one third of customers register and buy on the same day, and customers who did not make their first purchase in 30 days, tend to take a long time to complete. We also observed that the type of fuel and the use of the discount influence the time until the first purchase. Other customer variables can be used to deepen the characterization of the time until the first purchase with Shell Box and 
Table 9. Merged table data.

\begin{tabular}{ccccccc}
\hline store_id & shell_box & total & ratio_c & ratio_r & location & shell_box_rate \\
\hline 636800 & 7922 & 89553 & 0.088 & 0.097 & {$[-21.18,-47.78]$} & 3 \\
1023970 & 1307 & 51068 & 0.025 & 0.055 & {$[-23.50,-47.45]$} & 2 \\
$\ldots$ & $\ldots$ & $\ldots$ & $\ldots$ & $\ldots$ & $\ldots$ & $\ldots$ \\
\hline
\end{tabular}

thus direct actions to obtain better results for the company. Another analysis that can be done is to identify factors that influence the time between two uses of the application, also with survival analysis, with which you could perform more assertive actions to retain the customer.

\section{WP2 - Visual Data Exploration}

On the visualization approach, the main problem addressed was to build a pipeline in which the given data were processed and enriched in order to map each important variable to a specific visual domain. The activities performed can be summarized as follows:

- Data processing to create a table ranked_stores.csv that unifies pumplogs.csv and stores.csv.

- Use of internet data to search for specific points of interest such as malls, schools, convenience stores, etc ... near each gas station in order to analyze the occupation in the surrounding environment.

- Create interactive maps in order to visualize the processed data. Among them we can cite folium for displaying maps, pandas for manipulation of tabular data and seaborn to plot charts.

\subsection{Data processing}

The data processing step consist in merging the tables pumplogs.csv and stores.csv in one major table named ranked stores.csv. The constructed table gather all information about the store and adds two more columns concerning the total number of transactions in a given gas station. The columns added were ratio_ $r$ and ratio_ $c$ defined as:

$$
\begin{gathered}
\text { ratio_c } c=\frac{\text { number of shell-box transactions in the gas station }}{\text { total of trasactions in the gas station }} \\
\text { ratio_r } r=\frac{\text { number of transactions in the gas station }}{\text { total of transactions in all gas stations }}
\end{gathered}
$$

Where the transactions cover the period from the beginning of 2019 to the end of 2020 .

Furthermore, we associated a discrete variable shell box rate that aims to divide the group of gas stations in three subsets in comparison with the mean value $m_{\text {rate }}$ and standard deviation $s_{\text {rate }}$ of shell_box_rate:

(1) Above average shell_box_rate : (greater than mean +1 standard deviation)

(2) Below average shell_box_rate : (lower than mean - 1 standard deviation) 


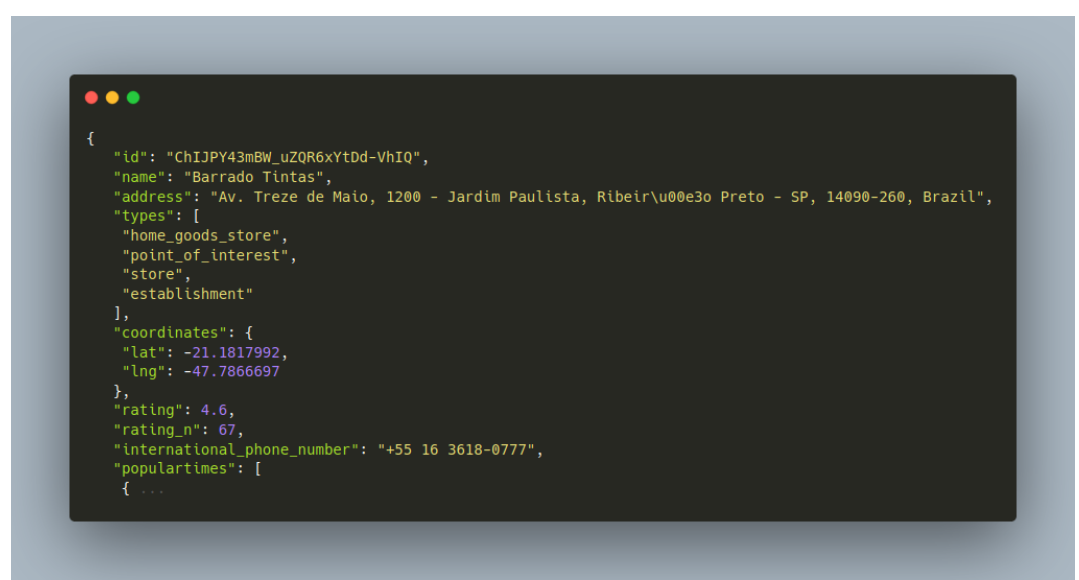

Figure 16. Example of returned data from the internet.

(3) In average shell_box_rate : otherwise

\subsection{Data acquisition}

Using data in the internet, we built an algorithm to proceed a search of all commercial business that are in a given range from a given geolocated point of interest, returning information about the business as, for instance, its name, geo coordinates and the type of the business as well, as shown below:

Considering the 10 gas stations with the best ratio score and the 10 gas stations with the worst ratio score and a range of $500 \mathrm{~m}$, this algorithm returned 2502 business, on an average of 60 business near each gas station.

In the next step, we built a filter to select only business that may have bigger impact on vehicle usage. The types of these business is an extensive list that includes business as: ATM, bank, bar,car dealer, car rental, car repair,car wash,church, city hall, convenience store, courthouse, department store,drugstore, electronics store, food, furniture, store, grocery or supermarket, gym, home goods store, hospital,laundry, liquor store, local government office, lodging, meal delivery, meal takeaway, moving company, night club, parking, pharmacy, place of worship, police, primary school, restaurant, school, secondary school, shopping mall, stadium, store, supermarket, taxi stand and university.

This reduced the set of business to 483 places. Using web scraping techniques, we obtained the Google's hourly occupancy score data for each place considered (score available on the google maps website).

\subsection{Data visualization}

After processing of the occupancy score data, we were able to analyze the flow of people (with probable use of vehicles) near each gas station and we produce a dynamical visualization through a heat map for each region of each city at a specific time of the day, together with a list of more used business in the region. The map was generated for 


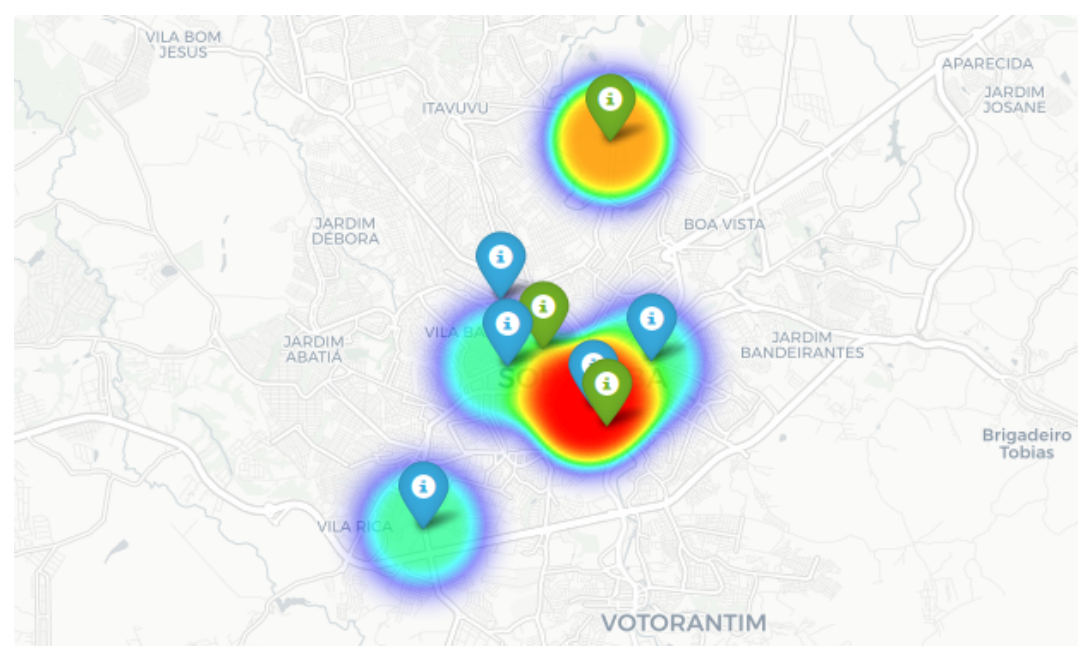

Figure 17. Example of heatmap generated.

Table 10. Most occupied business nearby.

\begin{tabular}{ccc}
\hline & Name & Occupation Score \\
\hline 1 & Fran Imports & 54 \\
2 & Linc Conveniência e Padaria & 45 \\
3 & Shell Select Convenience & 38 \\
$\ldots$ & $\ldots$ & $\ldots$ \\
\hline
\end{tabular}

the gas stations with the best and worst shell box performance. Here is a sample of the resulting visualization:

This type of information could be integrated with point-of-sale information (like sales, security cameras processing software [5] etc) in order to offer a broader view of the conversion rate of the business.

\section{WP3 - Classical Pattern Recognition}

In this approach, we will analyze the intrinsic phenomena that exist in the presented database using two methodologies: the first one, described in Section 4.1, is dedicated to the visual recognition of gas station patterns, and the second one, detailed in Section 4.2 , is dedicated to handling a classification at gas stations to predict whether a given establishment is more likely to have a clientele that uses the Shell Box or not.

\subsection{VisGAS: a new visualization tool for Geographic Analysis of Stores}

For this approach, we collected information from all the presented databases involving information about consumers, transactions, fuel pumps, and stores to set up an exclusive database for gas stations, and for each station, several relevant information was added to the use of Shell Box App by customers. For example, we add information that describes the percentage of each fuel that is paid with shell box, how many shell box customers a 
station owns, what is the average number of stores that customers at a station visit, the average fuel price, the total number of customers, among others. However, to analyze all these characteristics together with the geographic and regional information of a post, we made a prototype of a visualization tool: VisGAS.

VisGAS, or "Visual Geographic Analysis of Stores", is a visual analytics system that facilitates analysis and integrates information about the gross, latent, and geographical characteristics of a set of gas stations. In Figure 18, the VisGAS prototype interface is presented, developed using the library Streamlit in Python. This visualization tool consists of four main visual components that are detailed in the sequence: $\mathbf{V C}_{1}, \mathbf{V C}_{2}$, $\mathbf{V C}_{3}$ and $\mathbf{V C}_{4}$.

$\mathbf{V C}_{1}$ : It is a filtering menu, represented on the left in Figure 18, which allows the specification of which characteristics should be analyzed. In detail, this component defines the filtering between the characteristics of the gas stations that will be addressed and how they will be presented in the other visual components. It also allows the definition of the local analysis of a single gas station.

$\mathbf{V C}_{2}$ : It is a two-dimensional plot, represented in the top mid of Figure 18, which represents the projection of the gas stations considered. Indeed, it is possible to define two types of plots:

- Scatter Plot: In this case, the gas stations are designed in such a way that the two axes of the plot represent two distinct features of each station, which can be defined in the component $\mathbf{V C}_{1}$.

- Singular Value Decomposition (SVD): In this case, gas stations are designed according to the two components with the greatest variance of their SVD. That is, the stations are projected according to their coordinates of the latent space $\mathbb{R}^{2}$.

Also, it is possible to define two more configurations of this component through $\mathbf{V C}_{1}$ : the radius and the color of the circle that represents the projection of each station. Both configurations are obtained using a continuous scale function and must represent two distinct features of the database that makes up the information table of gas stations. Besides, the color of the circles can be defined according to the labels they receive in a grouping process using the technique $K$-Means [6] with $K=4$, and in the case of SVD projection, the process is conducted directly in the coordinates of the stations in the latent space.

$\mathbf{V C}_{3}$ : This component, represented in the top right of Figure 18, is defined by a map plot that allows geographic analysis to be carried out around a specific gas station. Its main intention is to facilitate the detection of logistical structures, such as highways, urban conglomerates, parks, etc., close to the analyzed station.

$\mathbf{V C}_{4}$ : In our prototype, this component is the only static component of the tool and is located at the bottom of Figure 18, consisting of the presentation of an image of the front of the analyzed station. In the future, the intention is that this component will become an interactive widget such as Google Street View. 


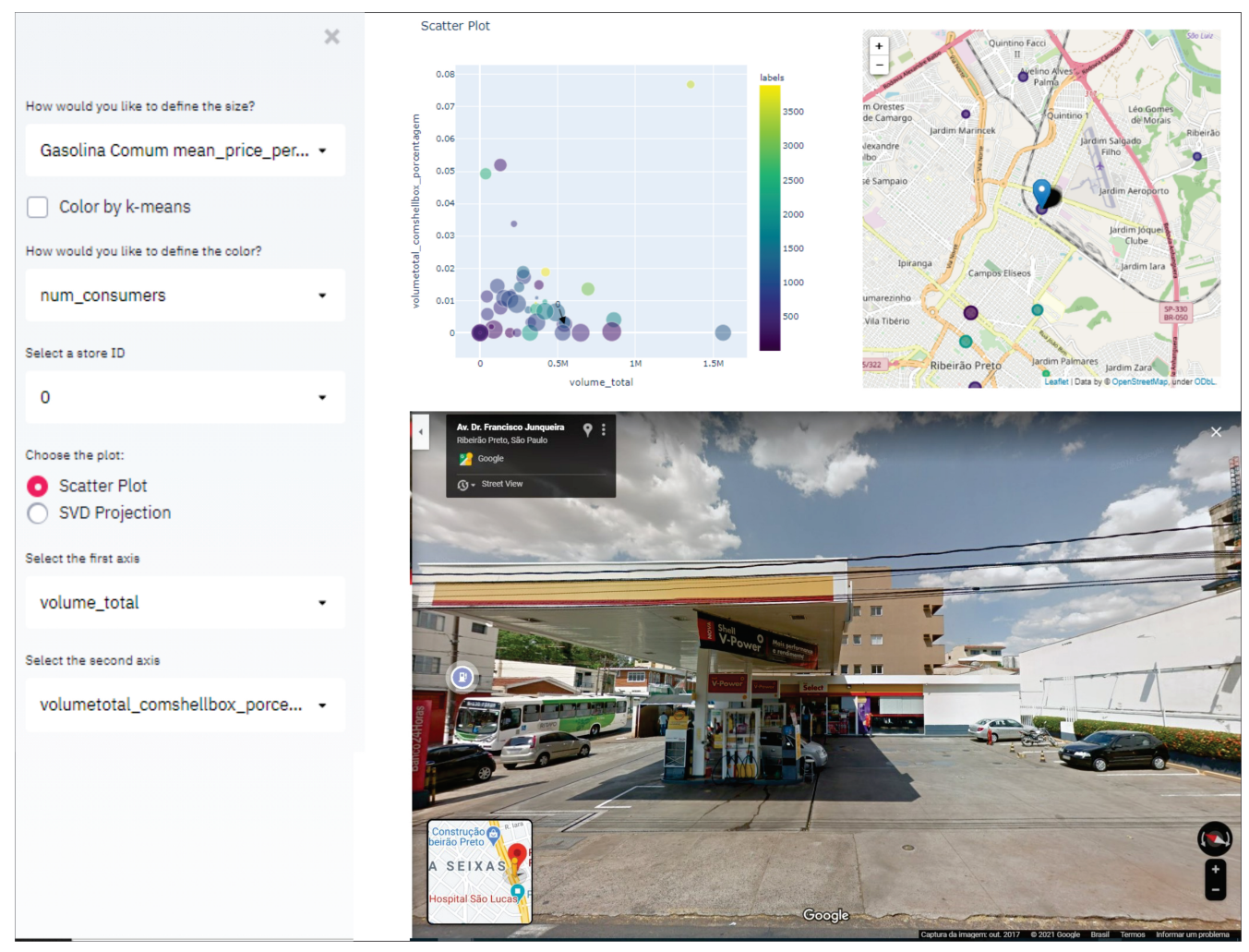

Figure 18. VisGAS prototype interface.

\subsubsection{Case Study}

We evaluated VisGAS's ability to summarize information in a case study. In this environment, two main questions were raised that we try to answer using the developed system. These are elaborated below:

$\mathbf{Q}_{1}$ : "Is there segregation that organizes the set of gas stations into several groups with different features?".

$\mathbf{Q}_{2}$ : "If $\mathbf{Q}_{1}$ is answered in the affirmative, what would be defining the segregation of the stations? How does each service influence the adherence to the Shell Box in its respective clientele? Would this be associated with an urban phenomenon, such as the location of the station, monetary, such as the price of fuel adopted, or even simply numerical, as if the adhesion of Shell Box was proportional to the total number of customers?".

To answer $\mathbf{Q}_{1}$, let's look at the set of stores in its latent space. Thus, given the matrix of stores $\mathbf{S}$, according to Equation (4.1), in which each column represents information from the same station, we will map each station in a latent space of only three dimensions making use of SVD. 


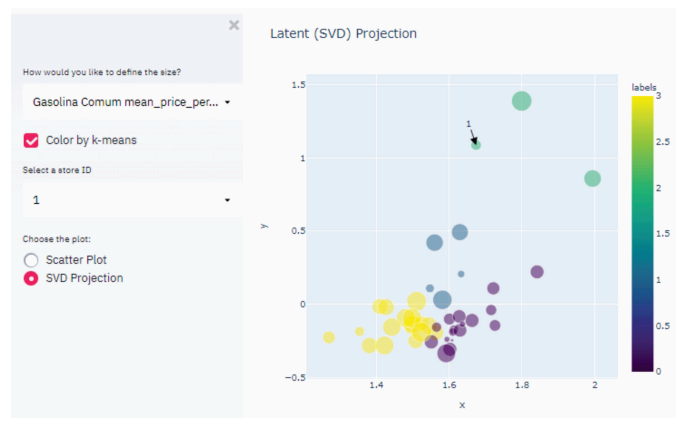

Figure 19. SVD projection and K-means classification.

$$
\mathbf{S}=\left[\begin{array}{cccc}
\mid & \mid & \cdots & \mid \\
s_{1} & s_{2} & \cdots & s_{n} \\
\mid & \mid & \cdots & \mid
\end{array}\right]
$$

where $n$ is the total number of stores.

Next, we will run a clustering algorithm (K-means) that will segregate the representations of the posts in the latent space into four distinct groups. For visual purposes, we will design these posts considering their two components of the latent space with the greatest variance and we will color these posts according to the resulting classification of K-means. VisGAS allows the conduction of this script if we configure the component $\mathbf{V C}_{1}$ as shown in Figure 19, that is, selecting the options "SVD Projection" and "Color by k-means". It is clear from the image that there are four well-defined groupings and that this phenomenon has little correlation with certain features of each station, such as the average price of common gasoline, which is represented by the radius of the circles, since there is a smaller circle and there is a bigger circle in practically all the groups generated. This phenomenon occurs with all variables associated with gas stations. In this way, we can answer the question $\mathbf{Q}_{1}$ by pointing out that there are well-defined groups of gas stations, which characterizes that, indeed, there is a combination of factors that differentiate them. However, further analysis must be conducted in order to indicate what specifically is segregating the set of stations.

The question $\mathbf{Q}_{2}$ deals with using VisGAS to understand why the good segregation obtained in $\mathbf{Q}_{1}$. In other words, to answer the second question, we will have to use all the components of VisGAS concurrently. Immediately, we will use the component $\mathbf{V C}_{1}$ so that it is possible to visualize in the scatter plot, arranged in the component $\mathbf{V C}_{2}$, the influence of the four most important features: total volume sold, defining the first axis; percentage of volume sold with shell box, defining the second axis; the total number of the gas station customers, defining the color; and the average price of gasoline, defining the radius, in which the bigger the more expensive the product is. As a result, we have the graph shown in Figure 20.

When analyzing the scatter plot, we noticed that most of the stations in this base remain in the lower left quadrant of the plot, which means that a small number of establishments sell a lot of fuel or have a high sales rate with Shell Box. To meet $\mathbf{Q}_{2}$, we will focus our analysis on four of these posts, which are on the margins of the main 


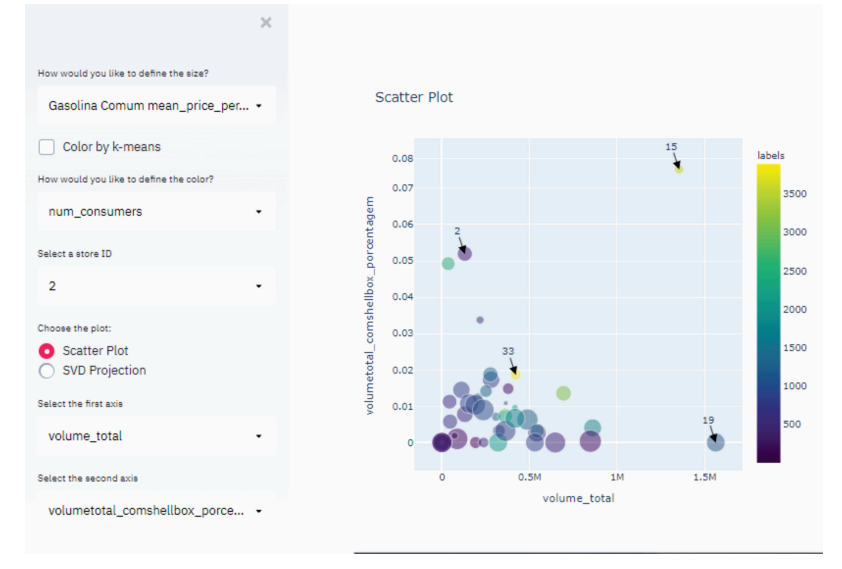

Figure 20. Scatter plot considering as axes the total volume sold versus volume sold with shell box. The color of the circle is defined by the total number of the gas station customers and the size is defined by the average price of gasoline sold at that gas station.

concentration of the chart, these being the stores with index 2, 15, 19, and 33. Of these stations, the ones that have a high sales rate with Shell Box are the index posts 2 and 15. However, no variable analyzed in the scatter plot is capable of being associated with this success. In detail, we can see that the station with the greatest use of the application in fuel sales is the station 15 , which is one of the stations with the largest number of consumers, second only to the station 33 which is well below it in the chart and, therefore, has a lower sales rate with the application. It is also worth noting that the average price of gasoline does not define the degree of adherence, both when comparing these two agents and when comparing the 2 and 19 service stations. In this case, these two have an average price of gasoline and a similar number of consumers because they are of similar color and size. However, the station 19, whose customers use the application less, sells much more gasoline, even though it is one of the stations with the highest average price in the comparison. Therefore, we conclude that no numerical or monetary factor is defining the success rate of implementation of the application in payment, which may serve as an indication that the phenomenon occurs according to the geographical characteristics of the post. For this, we started to analyze on the map, described in the component $\mathbf{V C}_{3}$, the geographic surroundings of each station in search of more information, as highlighted in Figure 21.

The neighborhood of each gas station shown on the maps appears to be associated with the number of customers at the same gas station. In detail, the neighborhood of the store 15 (Figure 21(a)) is very similar to the neighborhood of the store 33 (Figure 21(b)), which, according to the scatter plot, have a similar number of customers. These stations are located on a busy avenue and are located between a residential complex and an industrial complex. In the same way, we can see that both stations 2 (Figure 21(c)) and 19 (Figure 21(d)) are located on a highway that gives access to a residential complex. However, the clientele of the pairs of stations compared has a very different rate of adherence to the application described, which indicates that the location of the station is also not very associated with the successful use of the Shell Box. Thus, to complete 


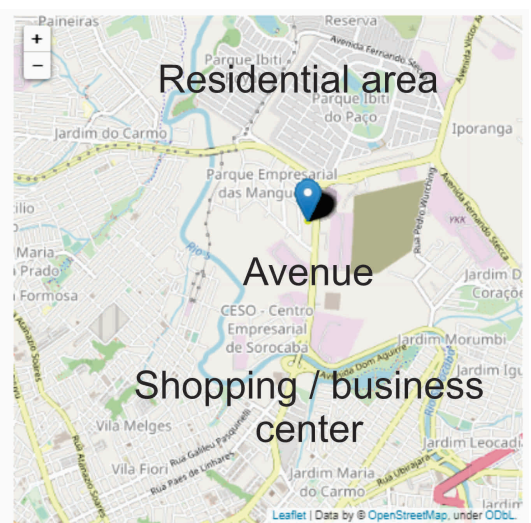

(a) Gas station 15

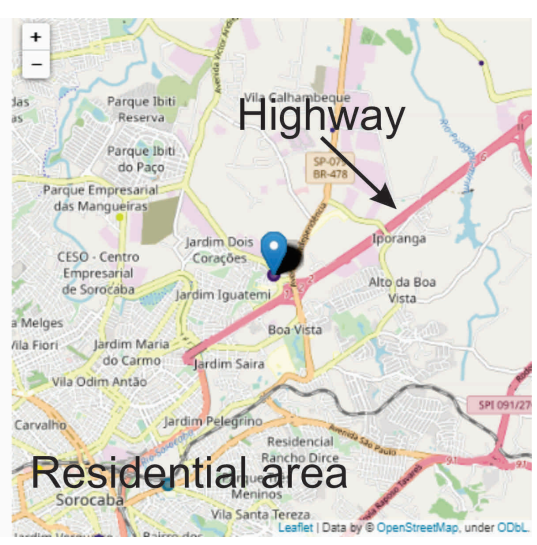

(c) Gas station 2

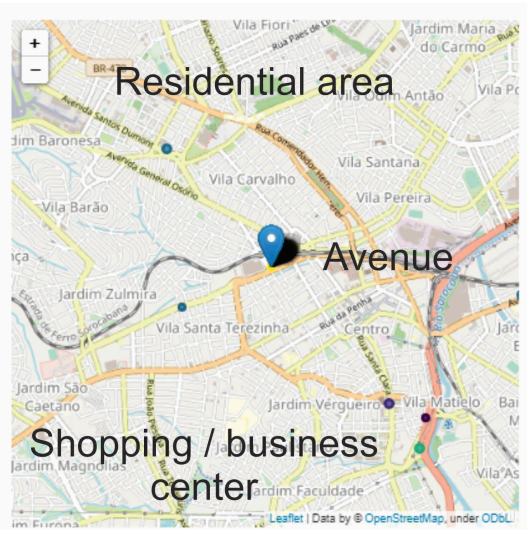

(b) Gas station 33

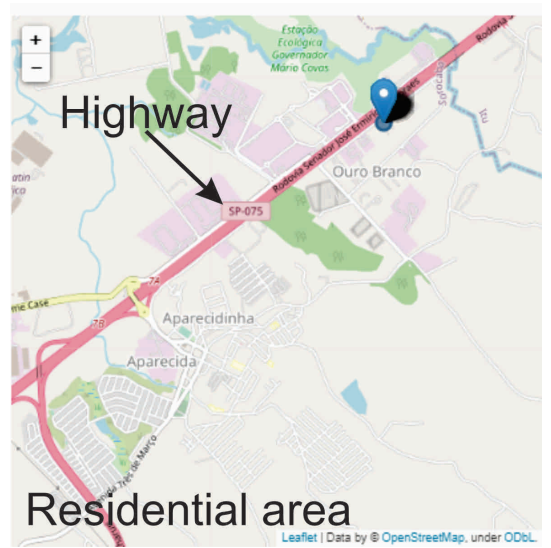

(d) Gas station 19

Figure 21. Maps of the gas stations evaluated on the scatter plot and their respective highlighted urban features.

the answer to the question $\mathbf{Q}_{2}$, we will use the component $\mathbf{F}_{4}$ and analyze the front of the gas stations considered, which are presented in Figure 22.

Our intention in using the component $\mathbf{V C}_{4}$ is to detect if there is any structural and visual aspect present in the construction of the gas station that defines its type of clientele and, consequently, defines the use of Shell Box. Indeed, it seems to be a trend that gas stations with a larger commercial area and with pumps further away tend to have greater adherence to the Shell Box, as is the case of the stations 15 (Figure 22(a)) and 2 (Figure $22(\mathrm{c}))$. This phenomenon can also be observed in the other gas stations with proportional payment rate with the application greater than $0.03 \%$, which is the case of the stations 12 (Figure 22(e)) and 42 ( Figure 22(f)). At the same time, gas stations that are located in more limited locations and with a smaller commercial area tend to have a clientele that makes less use of the application, as is the case with the considered stations 33 (Figure 22(b)) and 19 (Figure 22(d)). In addition, the other stations located further down on the scatter plot and which, therefore, have a clientele with less adherence to the application, 


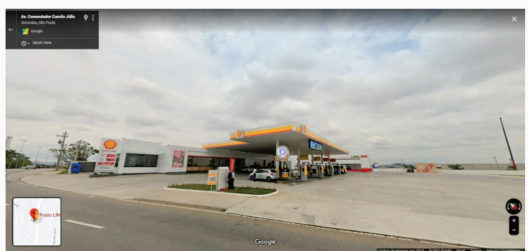

(a) Gas station 15

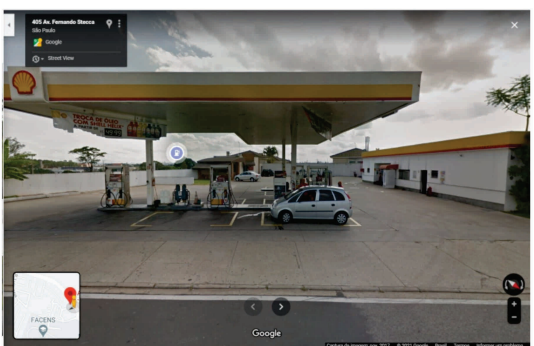

(c) Gas station 2

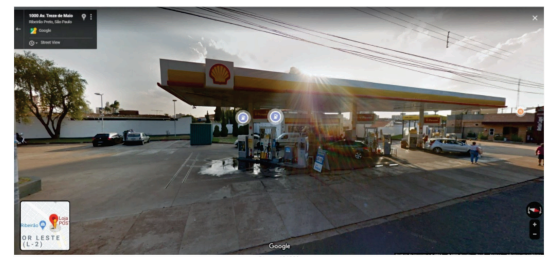

(e) Gas station 12

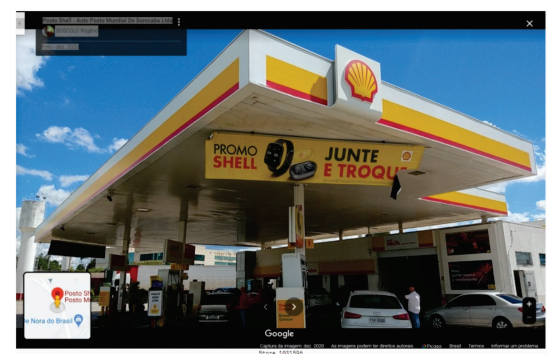

(g) Gas station 30

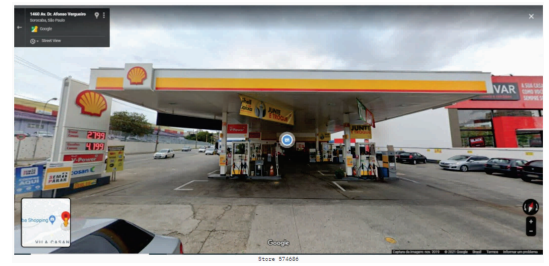

(b) Gas station 33

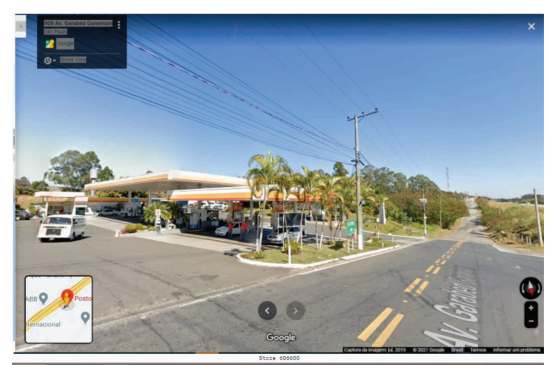

(d) Gas station 19

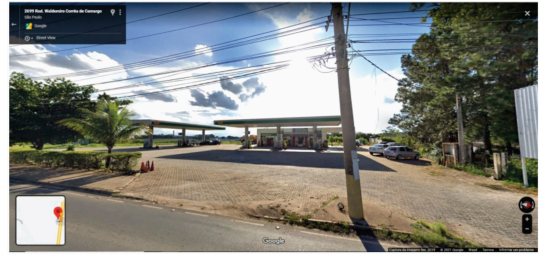

(f) Gas station 42

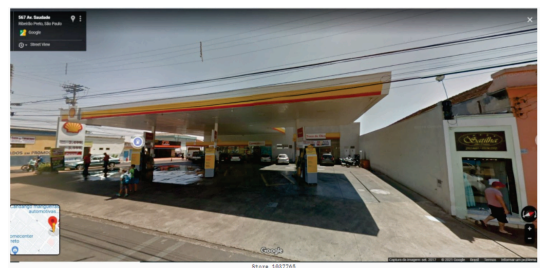

(h) Gas station 5

Figure 22. Front of the gas stations evaluated on the scatter plot.

also have a more limited front and with less area. In this case, we can mention as an example, the stores 30 (Figure 22(g)) and 5 (Figure 22(h)).

\subsection{ShellBox Conversion Rate Prediction}

We also investigated whether it is feasible to exploit supervised learning approaches to build classifiers. We focused on the problem of developing models capable of predicting the gas station ShellBox conversion rate (SCR) of a specific gas station. The first step 
was a combination of feature engineering, using features provided in the Raizen dataset; and an enrichment of the dataset, by incorporating demographic information.

In the feature engineering step, we first investigated which gas station features could be relevant to predict conversion rate. We hypothesized that the sales prevalence for a specific fuel type could be related to the ShellBox conversion rate. Thus, we designed the following features:

$$
\begin{aligned}
\text { features: }_{\text {gasoline }} & =\frac{\text { gasoline }_{\mathrm{vol}}}{\text { gasoline }_{\mathrm{vol}}+\text { ethanol }_{\mathrm{vol}}+\text { diesel }_{\mathrm{vol}}+\text { gnv }_{\mathrm{vol}}}, \\
\text { ethanol }_{\text {norm }} & =\frac{\text { ethanol }_{\mathrm{vol}}}{\text { gasoline }_{\mathrm{vol}}+\text { ethanol }_{\mathrm{vol}}+\operatorname{diesel}_{\mathrm{vol}}+\text { gnv }_{\mathrm{vol}}}, \\
\text { diesel }_{\mathrm{norm}} & =\frac{\text { diesel }_{\mathrm{vol}}}{\text { gasoline }_{\mathrm{vol}}+\text { ethanol }_{\mathrm{vol}}+\text { diesel }_{\mathrm{vol}}+\text { gnv }_{\mathrm{vol}}}, \\
\text { gnv }_{\text {norm }} & =\frac{\text { gnv }_{\mathrm{vol}}}{\text { gasoline }_{\mathrm{vol}}+\text { ethanol }_{\mathrm{vol}}+\text { diesel }_{\mathrm{vol}}+\text { gnv }_{\mathrm{vol}}},
\end{aligned}
$$

which is essentially a normalization of fuel sales volume by fuel type. Note that specific types of fuel were grouped in these four main fuel types.

However, a preliminary visual exploration revealed that the aforementioned features were not sufficiently descriptive for the studied prediction problem. We next enriched the gas station dataset with demographic information, by exploiting the location of each gas station (which was included in the original dataset). The following information was included:

- populational density in the neighborhood;

- average income in the neighborhood;

- consumption potential in the neighborhood.

Finally, the input representation of each gas station was defined as a vector in a feature space of dimension 7 .

By employing the well-known Multidimensional scaling (MDS) projection method to the feature vectors, and encoding the SCR in the color of each projected sample, we could identify patterns related to high potential for ShellBox conversion in the data. As shown in Figure 23, gas stations with higher SCR seem to occur in the center of the plot. This encouraged us to develop the proposed classifier.

We performed a binary labeling of this dataset (represented by the engineered feature vectors) as high or low SCR potential, by simply thresholding the SCR, as visually shown in Figure 24.

Binary SVM classifiers with Gaussian kernel were adopted. We performed the wellknown $k$-fold cross-validation with $k=5$ folds, to tune the hyperparameters $\gamma$ and $C$ through a grid search in the bidimensional hyperparameters space.

The best classifiers achieved up to $80 \%$ of accuracy. Given the small size and the poor availability of relevant features in the dataset, we considered that this was an encouraging result. Figure 25, visually reveals the results of the best classifier in a specific validation fold. Gas stations with low ShellBox conversion potential are represented as squares, while high potential gas stations are represented as circles. The training data is encoded in blue colors; correctly predicted validation data in green, and incorrectly predicted in 


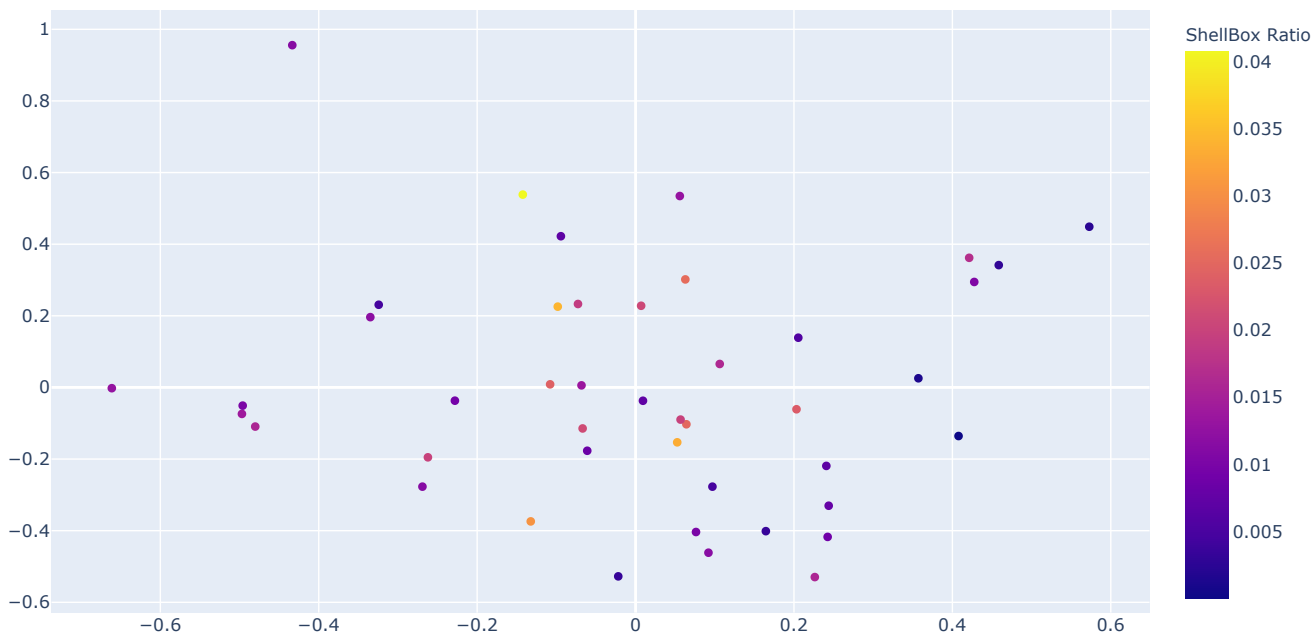

Figure 23. MDS projection of gas stations' feature vectors with SCR encoded in colors: a region of higher SCR seems to exists.

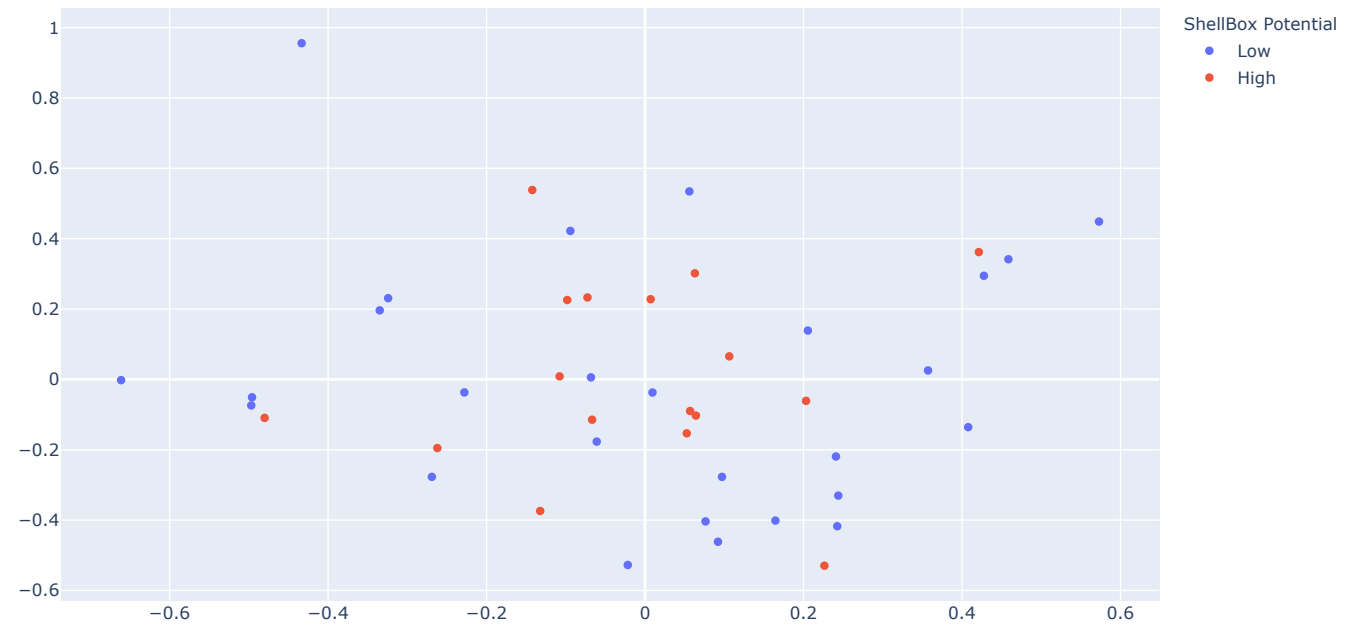

Figure 24. Binary labeling of the dataset as high or low potential for ShellBox conversion.

red. In the center of the plot, one can see a red square, which may indicate a gas station with a high potential for ShellBox conversion may have an issue. Thus, misclassifications may give insight for further investigation of gas stations.

\section{WP4 - Analysis of User Reviews}

Our goal in the section is prototype tools that may be be used to analyse the user experience in the app, as well as the text interaction between the user and the app support crew, providing insights and answers for improvements in the app, marketing campaigns efficiency, performance of sales among other managing challenges.

Now we describe the pipeline that we used to analyzing the user experience of the App 


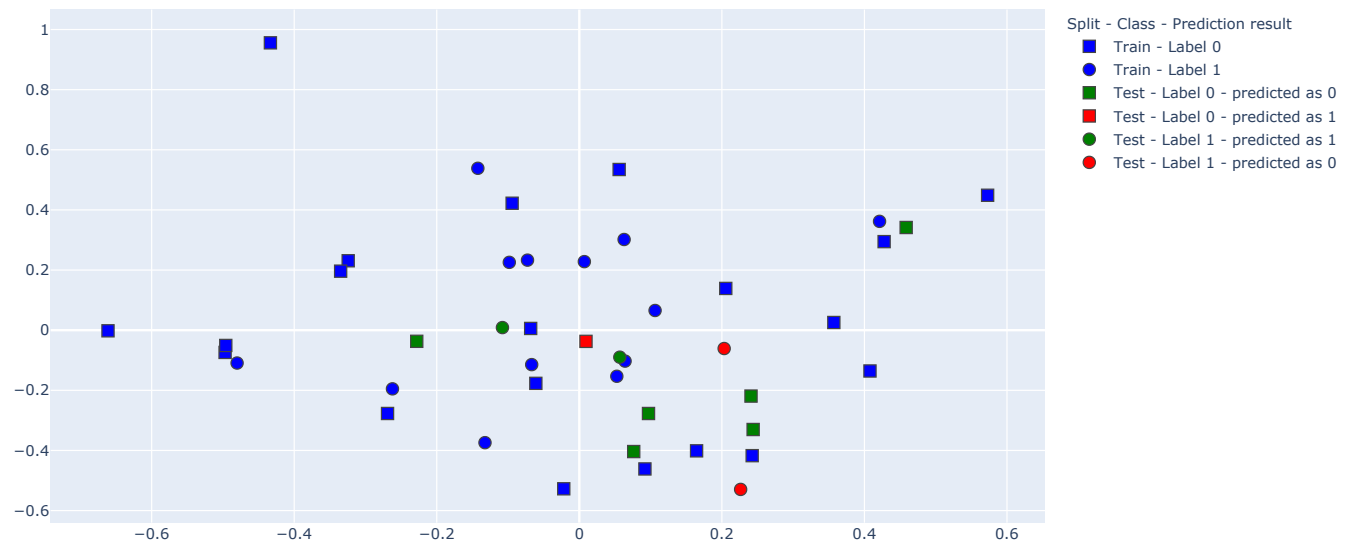

Figure 25. SCR prediction results in a specific fold of the best classifier.

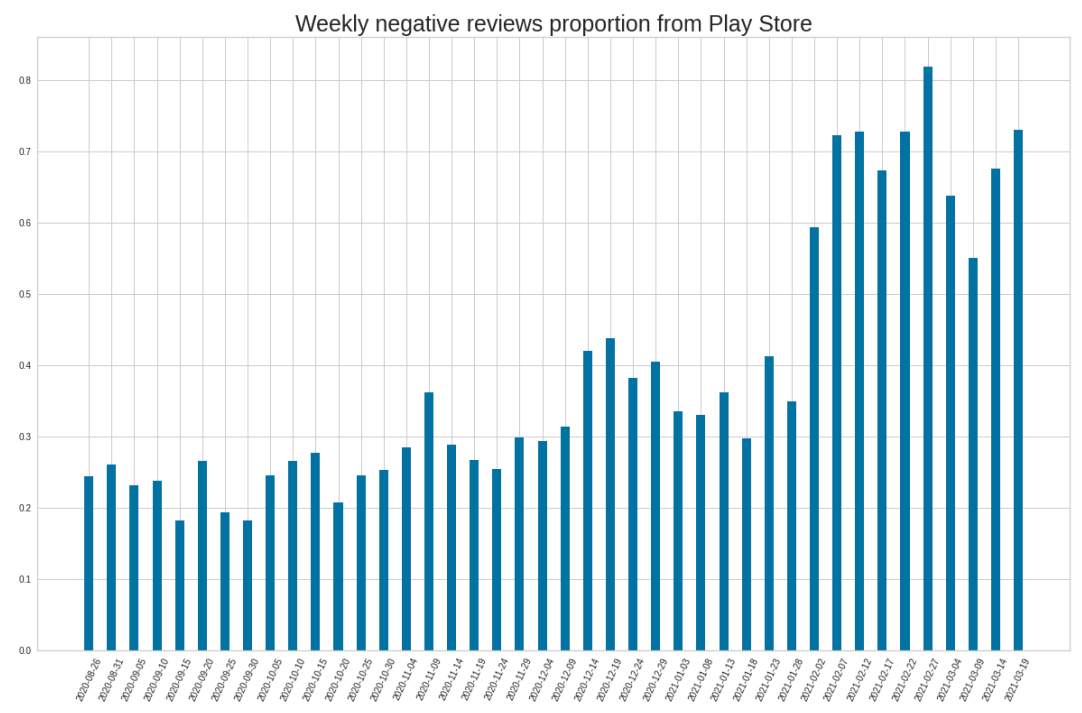

Figure 26. Visualization and analysis of reviews from Play Store

Shell Box. We started our analysis collecting data from user reviews in the Apple Store, Google Play Store and Instagram. In a future project, we could analyze data from other social medias such as: YouTube, Twitter, Facebook and Instagram and integrate them.

\subsection{Web Scraping of users' reviews}

Using web scrapping techniques, we collected 2897 Apple user's reviews from App Store and 12240 user's reviews from Play Store. Analyzing Play Store reviews as shown in figure 26 , we can see that the negative rating reviews (1 and 2 stars) increases along the weeks, which indicates that the app is not performing well on the user's side.

Next, we use Artificial Intelligence and Natural Language Processing tools to transform each review or response in a mathematical object, a vector in a space of dimension around 
Play store reviews colored by their rating

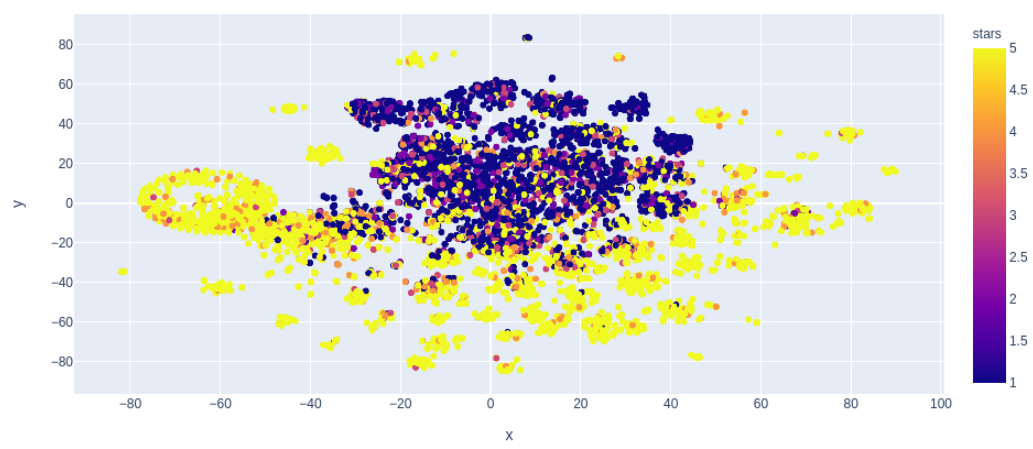

Figure 27. Play store reviews colored by their rating

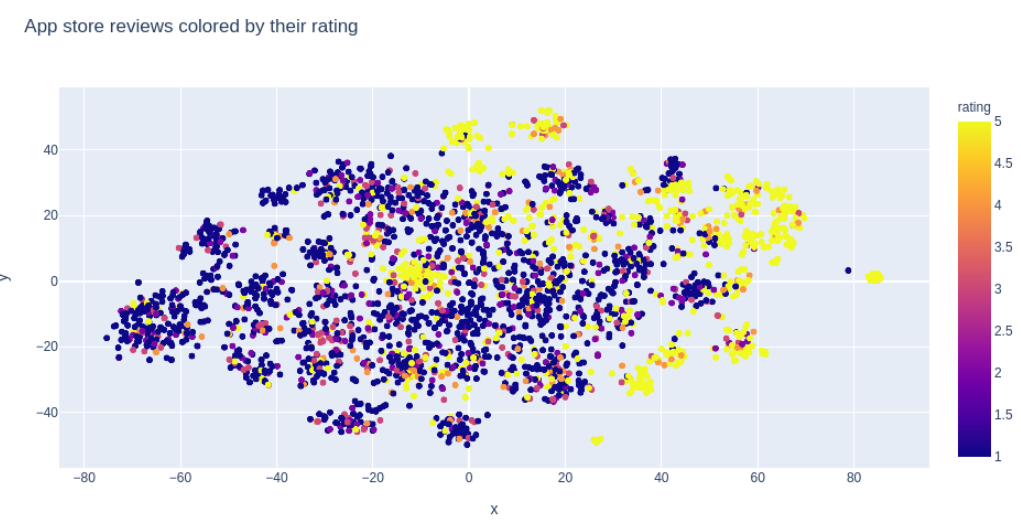

Figure 28. App store reviews colored by their rating

3000 , the size of the corpus associated to the reviews. We use the Singular Decomposition Value Theorem, a classical technique also used in section WP3, to reduce the dimension 3000 to dimension 50, in order to plot points in the plane using t-SNE projections. This may give us clusters of reviews (or answers) As shown in the picture 27. This visualization tends to group reviews with a similar semantics, clustering reviews that are related to similar problems or positive feedbacks.

The colors represent the rating score of the review given by the user, from 1 (yellow) to 5 (blue). Each review is represented by a colored dot. It is clear that this approach may be extrapolated to a weekly analysis, giving a (almost) real time view of the problems in the app reported by the reviews.

Similarly, the figure 28 shows a visualization of the reviews from Apple Store, in this case we can see a higher proportion and scattered negative reviews in the plot.

We also plotted the developer answers of the reviews and we colored them accordingly to the review's rating associated with the developer answer, as shown in the figure 29. 


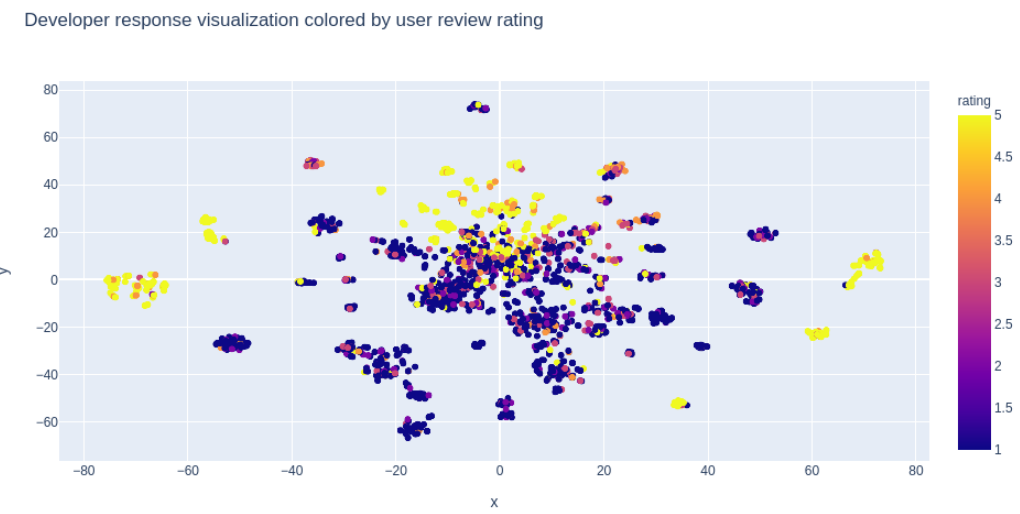

Figure 29. Visualization of developer responses colored by review rating

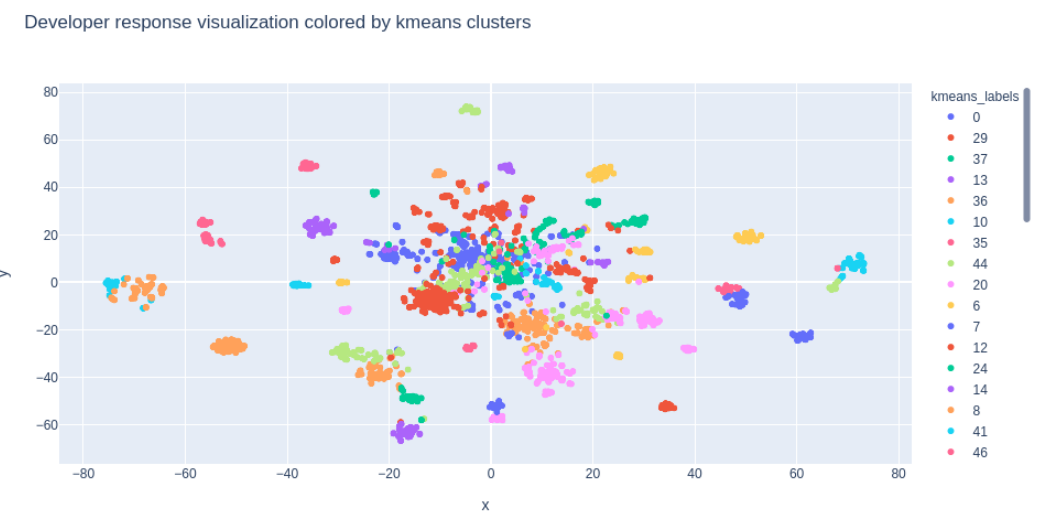

Figure 30. Visualization of developer responses colored by kmeans clusterization

In the figure 30, we run the clusterization algorithm k-means and we colored the answers accordingly to the cluster label.

After all, we plotted the word cloud of each cluster as following: for each cluster, we made a word cloud for the reviews and another for the developer response, them we measured the rating mean of each cluster, getting the following results as shown in the figures 31 and 32 .

\section{Conclusion and Future Directions}

As future research directions, we can cite further explorations related to data classification through machine learning approaches. With machine learning, we can classify the Shell stores according to their primary service features, such as location and gas types, and from this information, we can predict the probability of payment through the Shell Box App. In addition, we propose applying a new toolset that aims to understand and explain 

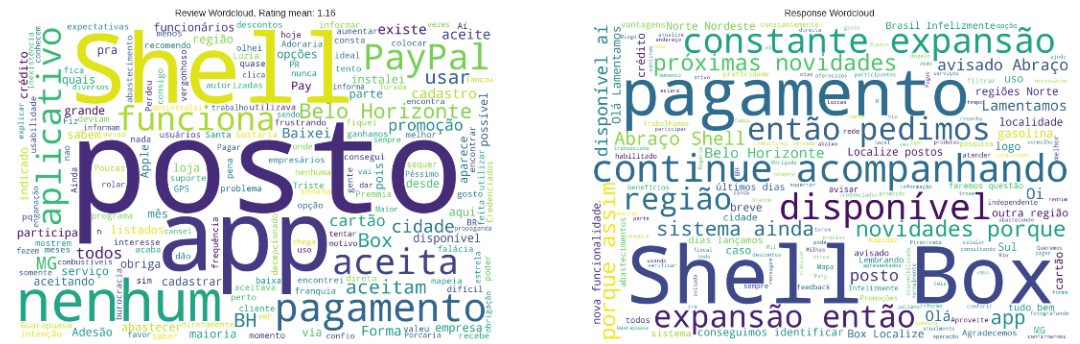

Figure 31. Left wordcloud shows user reviews and right wordcloud shows developer response for a given cluster with 1.16 rating mean
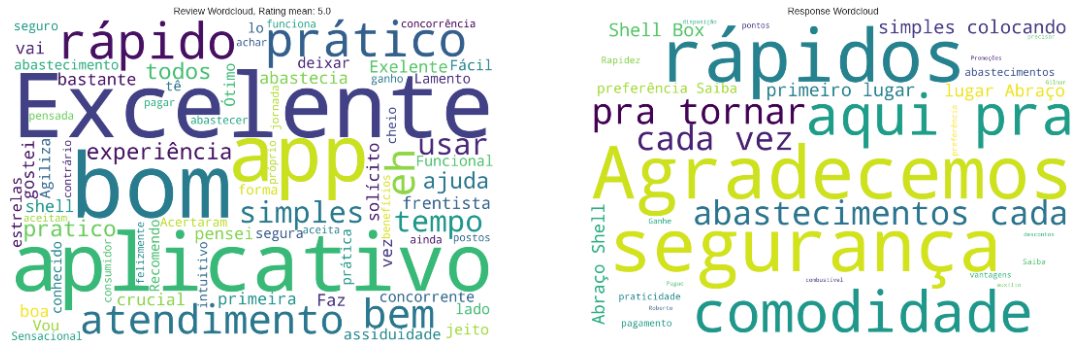

Figure 32. Left wordcloud shows user reviews and right wordcloud shows developer response for a given cluster with 5.0 rating mean

the reasons behind predictions, the Explainable Machine Learning. Therefore, with these learning approaches, we can demonstrate the probability of a Shell gas station convert its sales into Shell Box App and explain the main reasons for that probability. A pretty important feature in assessing trust is fundamental if Shell plans to take actions related to Shell stores layout, marketing campaigns, or even staff training based on Shell Box use data in its stations.

\section{References}

[1] T. W. Anderson. An introduction to multivariate analysis. New York: John Wiley, 2003.

[2] Douglas Bates, Martin Mächler, Ben Bolker, and Steve Walker. Fitting linear mixed-effects models using lme4. Journal of Statistical Software, 67(1):1-48, 2015.

[3] Enrico Antonio Colosimo and Suely Ruiz Giolo. Análise de sobrevivência aplicada. Editora Blucher, 2006.

[4] Andrew Gelman and Jennifer Hill. Data analysis using regression and multilevel/hierarchical models. Cambridge university press, 2006.

[5] Valerio Nogueira Jr., Hugo Oliveira, Jose Augusto Silva, Thales Vieira, and Krerley Oliveira. Retailnet: A deep learning approach for people counting and hot spots detection in retail stores. In 2019 32nd SIBGRAPI Conference on Graphics, Patterns and Images (SIBGRAPI), pages 155-162. IEEE, 2019.

[6] Aristidis Likas, Nikos Vlassis, and Jakob J Verbeek. The global k-means clustering algorithm. Pattern recognition, 36(2):451-461, 2003. 
[7] Andreas Wienke. Frailty models in survival analysis. CRC press, 2010. 